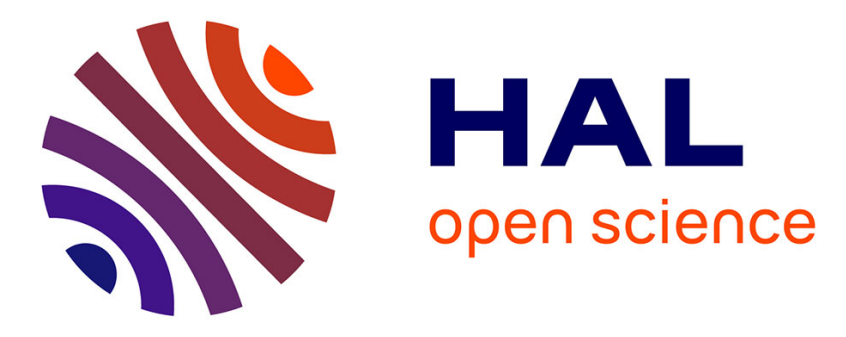

\title{
Hapke modeling of Rhea surface properties through Cassini-VIMS spectra
}

M. Ciarniello, F. Capaccioni, G. Filacchione, R.N. Clark, D.P. Cruikshank, P. Cerroni, A. Coradini, R.H. Brown, B.J. Buratti, F. Tosi, et al.

\section{- To cite this version:}

M. Ciarniello, F. Capaccioni, G. Filacchione, R.N. Clark, D.P. Cruikshank, et al.. Hapke modeling of Rhea surface properties through Cassini-VIMS spectra. Icarus, 2011, 214 (2), pp.541. 10.1016/j.icarus.2011.05.010 . hal-00786875

\section{HAL Id: hal-00786875 \\ https://hal.science/hal-00786875}

Submitted on 11 Feb 2013

HAL is a multi-disciplinary open access archive for the deposit and dissemination of scientific research documents, whether they are published or not. The documents may come from teaching and research institutions in France or abroad, or from public or private research centers.
L'archive ouverte pluridisciplinaire HAL, est destinée au dépôt et à la diffusion de documents scientifiques de niveau recherche, publiés ou non, émanant des établissements d'enseignement et de recherche français ou étrangers, des laboratoires publics ou privés. 


\section{Accepted Manuscript}

Hapke modeling of Rhea surface properties through Cassini-VIMS spectra

M. Ciarniello, F. Capaccioni, G. Filacchione, R.N. Clark, D.P. Cruikshank, P. Cerroni, A. Coradini, R.H. Brown, B.J. Buratti, F. Tosi, K. Stephan

PII:

S0019-1035(11)00175-8

DOI:

10.1016/j.icarus.2011.05.010

Reference:

YICAR 9817

To appear in:

Icarus

Received Date: $\quad 18$ January 2011

Revised Date: $\quad 9$ May 2011

Accepted Date: $\quad 10$ May 2011

Please cite this article as: Ciarniello, M., Capaccioni, F., Filacchione, G., Clark, R.N., Cruikshank, D.P., Cerroni, P., Coradini, A., Brown, R.H., Buratti, B.J., Tosi, F., Stephan, K., Hapke modeling of Rhea surface properties through Cassini-VIMS spectra, Icarus (2011), doi: 10.1016/j.icarus.2011.05.010

This is a PDF file of an unedited manuscript that has been accepted for publication. As a service to our customers we are providing this early version of the manuscript. The manuscript will undergo copyediting, typesetting, and review of the resulting proof before it is published in its final form. Please note that during the production process errors may be discovered which could affect the content, and all legal disclaimers that apply to the journal pertain. 


\section{Hapke modeling of Rhea surface properties through Cassini-VIMS}

\section{spectra}

M. Ciarniello $^{1 *}$, F. Capaccioni ${ }^{1}$, G. Filacchione ${ }^{1}$, R. N. Clark ${ }^{2}$, D. P. Cruikshank ${ }^{3}$, P. Cerroni ${ }^{1}$, A.

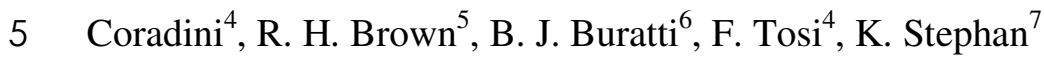

$7 \quad{ }^{1}$ INAF-IASF, via del Fosso del Cavaliere, 100, Rome, Italy

$8 \quad{ }^{2}$ U.S. Geological Survey, Federal Center, Denver, CO 80225 USA

$9 \quad{ }^{3}$ NASA Ames Research Center, Moffett Field, CA 94035 USA

$10{ }^{4}$ INAF-IFSI, via del Fosso del Cavaliere, 100, Rome, Italy

$11{ }^{5}$ Lunar and Planetary Lab and Steward Observatory, University of Arizona, Tucson, AZ 85721

12 USA

$13{ }^{6}$ Jet Propulsion Laboratory, California Institute of Technology, Pasadena, CA 91109 USA

$14 \quad{ }^{7}$ Institute for Planetary Exploration, DLR, Berlin, Germany 
*Corresponding author: Mauro Ciarniello

INAF-IASF Istituto di Astrofisica Spaziale e Fisica Cosmica

Via del Fosso del Cavaliere, 100, 00133 Rome, Italy

Phone: (0039)(06) 45488689

Email: mauro.ciarniello@iasf-roma.inaf.it

\section{Abstract}

The surface properties of the icy bodies in the saturnian system have been investigated by means of the CASSINI- VIMS (Visual Infrared Mapping Spectrometer) hyperspectral imager which operates in the $0.35-5.1 \mu \mathrm{m}$ wavelength range. In particular, we have analyzed 111 full disk hyperspectral images of Rhea ranging in solar phase between $0.08^{\circ}$ and $109.8^{\circ}$. These data have been previously analyzed by Filacchione et al. $(2007,2010)$ to study, adopting various "spectral indicators" (such as spectral slopes, band depth, continuum level, etc.), the relations among various saturnian satellites. As a further step we proceed in this paper to a quantitative evaluation of the physical parameters determining the spectrophotometric properties of Rhea's surface. To do this we have applied Hapke 
(1993) IMSA model (Isotropic Multiple Scattering Approximation) which allow us to model the phase function at VIS-IR (visible-infrared) wavelengths as well as the spectra taking into account various types of mixtures of surface materials. Thanks to this method we have been able to constrain the size of water ice particles covering the surface, the amount of organic contaminants, the large scale surface roughness and the opposition effect surge. From our analysis it appears that wavelength dependent parameters, e.g. opposition surge width $(h)$ and single-particle phase function parameters $(b, v)$, are strongly correlated to the estimated single-scattering albedo of particles. For Rhea the best fit solution is obtained by assuming:1) an intraparticle mixture of crystalline water ice and a small amount $(0.4 \%)$ of Triton tholin; 2$)$ a monodisperse grain size distribution having a particle diameter $a_{m}=38 \mu \mathrm{m}$; and 3) a surface roughness parameter value of $33^{\circ}$. The study of phase function shows that both Shadow Hiding and Coherent Backscattering contribute to the opposition surge. This study represents the first attempt, in the case of Rhea, to join the spectral and the photometric analysis. The surface model we derived gives a good quantitative description of both spectrum and phase curve of the satellite. The same approach and model, with appropriate modifications, shall be applied to VIMS data of the other icy satellites of Saturn, in order to reveal similarities and differences in the surface characteristics to understand how these bodies interact with their environment.

\section{Introduction and Rationale}

The Cassini spacecraft completed its initial four-year mission to explore the saturnian system in June 2008. Since then it entered in the extended mission phase (Cassini-Huygens Equinox Mission), which will last until September 2010. During all these years the VIMS (Visual and Infrared Mapping Spectrometer) instrument on board Cassini extensively observed the saturnian moons. The VIMS experiment consists of two imaging spectrometers observing the same field of view in two spectral ranges (Brown et al., 2004; McCord et al., 2004): VIMS-V spectrometer covering the 0.35 $1.05 \mu \mathrm{m}$ range in 96 spectral channels with a spectral sampling of $\Delta \lambda=7.3 \mathrm{~nm} / \mathrm{band}$ and spatial 
resolutions of $500 \times 500$ (nominal) or $166 \times 166$ (high resolution) $\mu \mathrm{rad} \times \mu \mathrm{rad} /$ pixel; VIMS-IR channel covering the $0.885-5.1 \mu \mathrm{m}$ range with 256 bands, a spectral sampling of $\Delta \lambda \approx 16 \mathrm{~nm} / \mathrm{band}$ and spatial resolutions of $500 \times 500$ (nominal) or $250 \times 500$ (high resolution) $\mu \mathrm{rad} \times \mu \mathrm{rad} /$ pixel (Miller et al., 1996).

VIMS collected both resolved and disk-integrated spectra of the moons in a wide range of observing conditions (solar phase angle and hemispheric coverage). While high spatial resolution observations of the satellites are essential to obtain compositional maps of the objects, the disk integrated observations are very useful to study the global properties of the surfaces and to point out correlations as well as differences among the various satellites.

The full disk observations constitute of a huge database with more than 1400 observations, obtained over a wide range of phase angles, for a total of 126000 spectra, covering the full VIMS spectral range. For a detailed description of the database see Filacchione et al. (2007, 2010) (in the following referred to as F2007 and F2010 respectively).

Spectrophotometry is a very powerful diagnostic tool in remote sensing to study the composition and the physical properties of the surfaces of objects under investigation. The amount of solar radiation, as a function of the wavelength, scattered from a surface towards the observer under varying observing conditions (incidence, emergence and phase angles) is a nonlinear function of several parameters such as the composition of the materials making up the surface, their grain size, the porosity and surface roughness of the interacting surface layers.

To retrieve quantitative information on these fundamental parameters of the surfaces, we need a multiple scattering model which provide approximate solutions to the radiative transfer in a particulate medium. The Hapke IMSA model (Hapke, 1993, 2002, 2008; Hapke et al. 2009) is one of several models developed to study the scattering properties of packed media (Lumme and Bowell, 1981; Drossart 1993; Shkuratov et al., 1999). It is an analytic two stream approximate 
solution to the radiative transfer equation and it has been applied successfully to perform photometric corrections of imaging data (Hudson and Ostro, 1999; Domingue et al., 2009), to investigate physical properties of regoliths (Mallama et al., 2002; Buratti et al., 2004) and to estimate surface compositions of planetary surfaces (Cruikshank et al., 2001; Cruikshank et al., 2005; Poulet et al., 2002).

In F2007 and F2010 the authors have adopted an empirical method of spectral analysis to reduce the dimensionality of the spectra by mapping high dimensional data into a lower dimension while preserving the main features of the original spectra. This led to the definition of a number of "Spectrophotometric Indicators" which are able to synthetically describe the spectrum. For instance spectral slopes in the visible range are a useful indicator of the degree of purity of water ice with respect to the presence of contaminants, thus two of the selected indicators are the slopes in the blue range of the spectrum (350-550nm) and in the NIR (near infrared) range (550-1000nm). In the IR range the most prominent features are the water ice absorption bands; consequently the authors have selected the depth of the 1.2, 1.5, 2.0 and 3.0 $\mu \mathrm{m}$ water ice bands as additional indicators.

The systematic analysis performed in F2007/F2010 on the basis of these indicators indeed pointed out several compositional trends within the satellites system and raised several questions which have not yet received a satisfactory answer. For instance:

- the correlation among Phoebe, Iapetus and Hyperion. The origin of the material that causes the albedo dichotomy of Iapetus has been the subject of a long standing debate (Buratti and Hicks, 2003; Spencer and Denk, 2010; Tosi et al., 2010). However, Clark et al. (2008, 2011b), showed that the visible colors and UV absorber are consistent with a single source with varying abundance of the contaminants. Key to solution of the problem was the discovery of Rayleigh scattering from small particles (Clark et al., 2008). From the VIMS 
data the spectral behaviour in the VIS-NIR range shows similarities between Iapetus and Hyperion; while the IR spectra point out a strong correlation between the features observed on Iapetus and Phoebe.

- The Band Depth at $1.5 \mu \mathrm{m}$ versus Band Depth at $2.0 \mu \mathrm{m}$ trend is the result of the combined effect of ice contamination, due to "darkening agents" as well as variable grain sizes, but the relative contribution of the two effects could not be ascertained. However, Clark et al. (2011a, 2011b) show that the 1.5 and $2.0 \mu \mathrm{m}$ ice band depth ratio is affected by the amount of sub-micron sized ice grains in the regolith.

- The symmetry of the 2-micron ice bands is unusual, being asymmetric toward longer wavelengths in spectra of the icy saturnian satellites (Clark et al., 2008) and in other icy objects (see review by Clark et al., 2011a). Clark et al., (2010b) showed that this asymmetry could be due to the presence of sub-micron ice grains and modelled the spectra using Hapke theory modified to include the diffraction component from those particles.

From this point of view the analysis performed in F2007 and F2010 represent a valuable empirical study to describe the global properties of the surfaces of the moons, however quantitative estimates on the nature (composition and physical properties) of the regolith require a full radiative transfer model. We have then set ourselves to work to this task (Ciarniello et al., 2010a, 2010b), and this paper describes the results we have obtained applying a radiative transfer model to the complete data set of Rhea's full disk observations.

Rhea, with a radius of $764 \pm 1.1 \mathrm{~km}$ (Thomas et al., 2006), is the second largest moon of Saturn; it orbits in the E ring with mean semimajor axis of $527070 \mathrm{~km}$ (see http://ssd.jpl.nasa.gov and reference therein); its mass is $\mathrm{M}=(2.306481 \pm 0.000059) \times 10^{21} \mathrm{~kg}$, which corresponds to a density 
142 of $1232.8 \pm 5.4 \mathrm{kgm}^{-3}$ (Iess et al., 2007) and geometric albedo of the satellite is 0.83 at $0.51 \mu \mathrm{m}$

143 (Pitman et al., 2010).

144 We have selected Rhea as the starting point for this analysis as it has the largest coverage in solar

145 phase angle and thus allows to thoroughly test the model. Our approach is based on a two steps

146 analysis: first a spectral fit is performed to retrieve the abundances of contaminants and ice grain

147 size, then these properties are used to compute single-scattering albedo at each wavelength, whose

148 values are adopted in the Rhea's phase function fit for the whole VIMS spectral range.

149 In section 2 we have described the method applied to model the phase curves and the spectra. In

150 section 3 the selected dataset is described as well as the reduction data procedure. In sections 4 and

1515 spectral and phase function fits respectively are analyzed. Section 6 concerns the feedback of

152 phase function fit to the spectrum fit. Conclusions and suggested future work are given in section 7.

\section{Hapke model}

157

158

159

160

161

162

163

164

Hapke's IMSA (Isotropic Multiple Scattering Approximation) model has been widely used to describe both solar phase curve and spectral properties of various objects in the solar system (Buratti 1985; Bowell et al. 1989; Domingue et al. 1995; Domingue and Verbiscer 1997). In this paper we refer to Hapke (1993) in order to describe the spectrophotometric properties of Rhea. For our analysis we have chosen full-disk images of the satellite and the formula we applied to describe the object full disk reflectance (FDR) as a function of the phase angle $g$ is straightforward derived by Eq. 10.40, p.275 in Hapke (1993):

$$
\begin{aligned}
& F D R(g)=\int_{A(i, v)} r(i, e, g) S(i, e, g) \mu d \Omega=\left\{\frac{1}{8}\left\{1-\sin \left(\frac{g}{2}\right) \tan \left(\frac{g}{2}\right) \ln \left[\cot \left(\frac{g}{4}\right)\right]\right\}\{(1+B(g)) p(g)-1] w+4 r_{0}\left(1-r_{0}\right)\right\}+ \\
& \left.+\frac{4}{3} r_{0}^{2}\left[\frac{\sin (g)+(\pi-g) \cos (g)}{2 \pi}\right]\right\} K(g, \bar{\theta})
\end{aligned}
$$


167 where $r_{0}=(1-\gamma) /(1+\gamma)$ and $\gamma=\sqrt{1-w}$.

168 The effect of porosity, which has been introduced in Hapke (2008), is not considered here, similarly

169 to Warell and Davidsson (2010), in order to reduce the number of free parameters in the following

170 inversion process. An improvement of the IMSA model is given in Hapke (2002). It provides a

171 more accurate analytic expression of the Ambartsumian-Chandrasekhar function $H(x)$ which

172 appears in the definition of the bidirectional reflectance $r(i, e, g)$. However, we adopted a linear

173 expression for $H(x)$ in the derivation of eq. 1 (eq. 8.56, p.212 in Hapke (1993)), since this

174 expression could be easily integrated. Eq. 1 represents the sum of the reflectances $r(i, e, g)$ of each

175 point on the surface $A(i, v)$, which is both viewed by the instrument and illuminated by the Sun, as a

176 function of the incidence $(i)$, emergence $(e)$ and phase $(g)$ angles. Each term is weighted by the

177 cosine of the emergence angle $\mu=\cos (e)$ which correctly projects the emitting area on the plane

178 orthogonal to the emission direction, and by the term $S(i, e, g)$ which describes the large scale surface

179 roughness (craters, depressions and other reliefs). Two mechanisms contribute to the emission

180 process: single scattering and multiple scattering. The first one depends on the single-particle phase

181 function $p(g)$, which describes how the light interacting with a particle is scattered. Actually the

182 single-particle phase function is an average on a small but statistically significant given volume of

183 particles. We modeled it assuming a double lobed Henyey-Greenstein (Henyey and Greenstein,

184 1941; Domingue and Verbiscer, 1997) phase function (Eq. 2) which depends on two parameters $b$

185 and $v$ : the first one describes the angular width of both forward and back scattering lobes, while the

186 second one describes their relative amplitude.

$187 \quad p(g)=\frac{1+v}{2} \frac{1-b^{2}}{\left(1-2 b \cos g+b^{2}\right)^{\frac{3}{2}}}+\frac{1-v}{2} \frac{1-b^{2}}{\left(1+2 b \cos g+b^{2}\right)^{\frac{3}{2}}}$ 
188

189

190

191

192

193

194

195

196

197

198

199

200

201

202

203

204

205

206

207

208

209

210

Another term which depends on single scattering is the one that accounts for the opposition effect $(\mathrm{OE}), B(g)$. It describes the observed non-linear increase in reflectance towards small phase angles.

In this work we use the mathematical formulation developed to treat shadow hiding opposition effect SHOE, which depends on the parameters $B_{0}$ and $h$, respectively the amplitude and the angular width of the effect. Rigorously, $B_{0}$ can assume values in the $0-1$ range, however we allowed $B_{0}$ to be greater than 1 in order to take into account coherent backscattering $(\mathrm{CB})$, which is another mechanism contributing to OE (Roush, 1994). We choose not to model explicitly the CBOE to reduce the number of free parameters. The term $K(g, \bar{\theta})$ in (Eq.1) is the full disk correction due to large scale surface roughness. Its value is always less than 1 and decreases with increasing roughness parameter $\bar{\theta}$, which is an average slope of the facets composing the surface.

Spectral information is included in the single-scattering albedo $w(\lambda)$. This parameter represents the fraction of light interacting with the particle (light can be absorbed, scattered or diffracted) that undergoes only scattering. It's value is in the range $0-1$ and depends on the medium optical constants $n$ and $k$ ( which are respectively the real and the imaginary part of the refractive index $m=n+i k)$, and on the grain size. Similarly to the case of single-particle phase function, the value of the single-scattering albedo is an average over a small but statistically significant volume of particles, and it is calculated as the ratio of the scattering and extinction efficiencies $Q_{S}$ and $Q_{E}$. In close-packed particulate media with spherical grains much larger than the wavelength (which is the assumption we made in our analysis) the IMSA model assumes that diffraction is negligible. In that case the extinction efficiency is 1 (the cross section of the particle is equal to the geometrical cross section) and single-scattering albedo is equal to the scattering efficiency $Q_{S}$, which can be directly calculated in the Hapke model once that optical constants of end-members, type of mixing and grain size are fixed. 
211 We have investigated three types of mixing: areal, intimate and intraparticle. Areal mixing (Fig. 1a)

212 is obtained averaging the reflectance of different patches of surface covered with different 213 materials:

$214 \quad r_{\text {tot }}=\sum_{i} p_{i} r_{i}$

215 where $r_{i}$ is the reflectance of $i$ th component and $p_{i}$ is the fraction of total surface covered.

216 Intimate mixing (Fig. 1b) describes a medium in which particles of different composition are mixed

217 together : this kind of mixing is obtained through a weighted average of single-scattering albedos of 218 the different types of grains:

$219 w_{\text {tot }}=\frac{\sum_{i} p_{i} \sigma_{i} w_{i}}{\sum_{i} p_{i} \sigma_{i}}$

where $\sigma_{i}$ is the geometrical cross section of the $i$ th particle type and $p_{i}$ is the volume percentage of 221 each component.

Intraparticle mixing (Fig. 1c) describes media in which inclusions of contaminants are embedded in a matrix of different optical properties. This kind of mixing is obtained with the Maxwell-Garnett rule (Maxwell-Garnett, 1904; Mallet et al.2005; Grundy, 2009):

$$
\varepsilon_{\text {eff }}=\varepsilon_{1}+3 \varepsilon_{1} p_{2} \frac{\left(\varepsilon_{2}-\varepsilon_{1}\right)}{\left[\varepsilon_{2}+2 \varepsilon_{1}-p_{2}\left(\varepsilon_{2}-\varepsilon_{1}\right)\right]}
$$

where $\varepsilon_{1}$ and $\varepsilon_{2}$ are the complex dielectric constants of the matrix and of the embedded material respectively, $p_{2}$ is the fraction of contaminant and $\varepsilon_{\text {eff }}$ is the effective complex dielectric constant of the particle. The dielectric constant is related to optical constants by the following relation: 


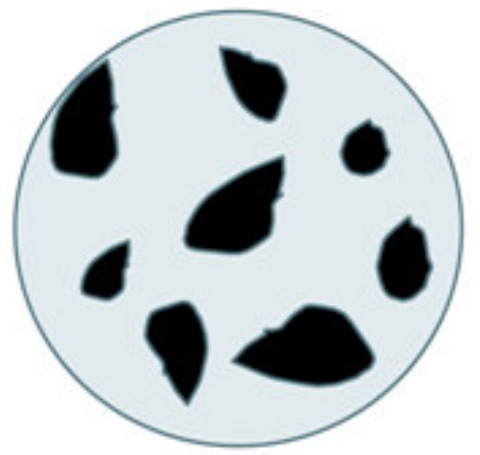

a)

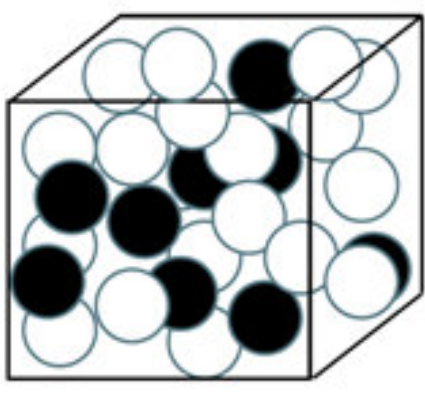

b)

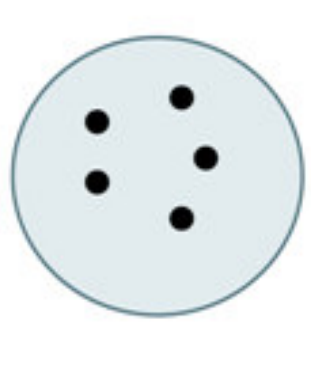

c)

[FIGURE 1]

\section{Observations and data reduction}

Our dataset is composed of 140 observations acquired by VIMS in the period January 2005 January 2008. Since Rhea exhibits a marked dichotomy between leading and trailing side (Verbiscer and Veverka, 1989; Buratti et al., 1998) we have selected only images in which the illuminated and observed area was more than $60 \%$ on the leading side, in order to study homogeneous regions of the satellite. With this limitation the total number of observations reduces to 111 with solar phase angles ranging from $0.08^{\circ}$ to $109.8^{\circ}$. both in normal and high-resolution modes. Accordingly, the satellite image size in the instrument 
246 field of view can cover from a few tens to some hundreds of pixels. In order to produce phase

247 function curves of the satellite at each wavelength we developed an IDL procedure that sums up the

248 reflectances $(\mathrm{I} / \mathrm{F})$ of observed-illuminated pixels in the image, correcting them by the multiplicative

249 factor $\delta \varepsilon D^{2} / R^{2}$ (where $R$ is Rhea's radius and $\delta \varepsilon$ is the solid angle subtended by the pixel). This

250 factor represents the solid angle increment on the satellite surface times the emission angle cosine.

251 The relation between observed data and FDR is:

252

$$
\int_{A(i, v)} r(i, e, g) S(i, e, g) \mu d \Omega \approx \sum_{j}\left(\frac{I}{F}\right)_{j} \frac{\delta \varepsilon D^{2}}{R^{2}}
$$

253 where the subscript $j$ identifies each single pixel.

254 In Fig. 2 Rhea full-disk normalized spectra acquired at various phase angle are plotted (for VIMS 255 calibration uncertainties please refer to McCord et al. (2004)). All the spectra exhibit typical 256 features of water ice (1.51,2.2 and 3.1 $\mu \mathrm{m}$ absorption bands), however towards the UV region the 257 shape of the spectrum strongly departs from the flat behavior of water ice producing a strong reddening. This feature has been traditionally assigned to the presence of organic contaminants as suggested by Cruikshank et al. (1998) and Poulet et al. (2002). However there is no clear additional signature in the IR, and this constrains the amount of contaminants to be at most few percent (Clark and Owensby, 1981). Clark et al., 2008 gave alternative explanations, including UV absorption by other compounds and very small grains (nano-phase) of opaque minerals such as hematite. Clark et al., (2011b) model the shape of the UV absorber with combinations of metallic iron (both large grained and nano-sized particles) and nano-phase hematite. A feature centered at $0.9 \mu \mathrm{m}$ is present in all the spectra. This seems to be an artifact due to the data calibration process. However, the presence of this feature does not affect the global slope in VIS-IR region and doesn't introduce any 267 offset between the two channels, and thus does not alter the results of the following analysis. The 268 spectra show a certain dependence on observing geometry. The slope in VIS-NIR (around $1 \mu \mathrm{m}$ ) 
270

271

272

273 function along the spectrum (even in this case related to $w$ ).

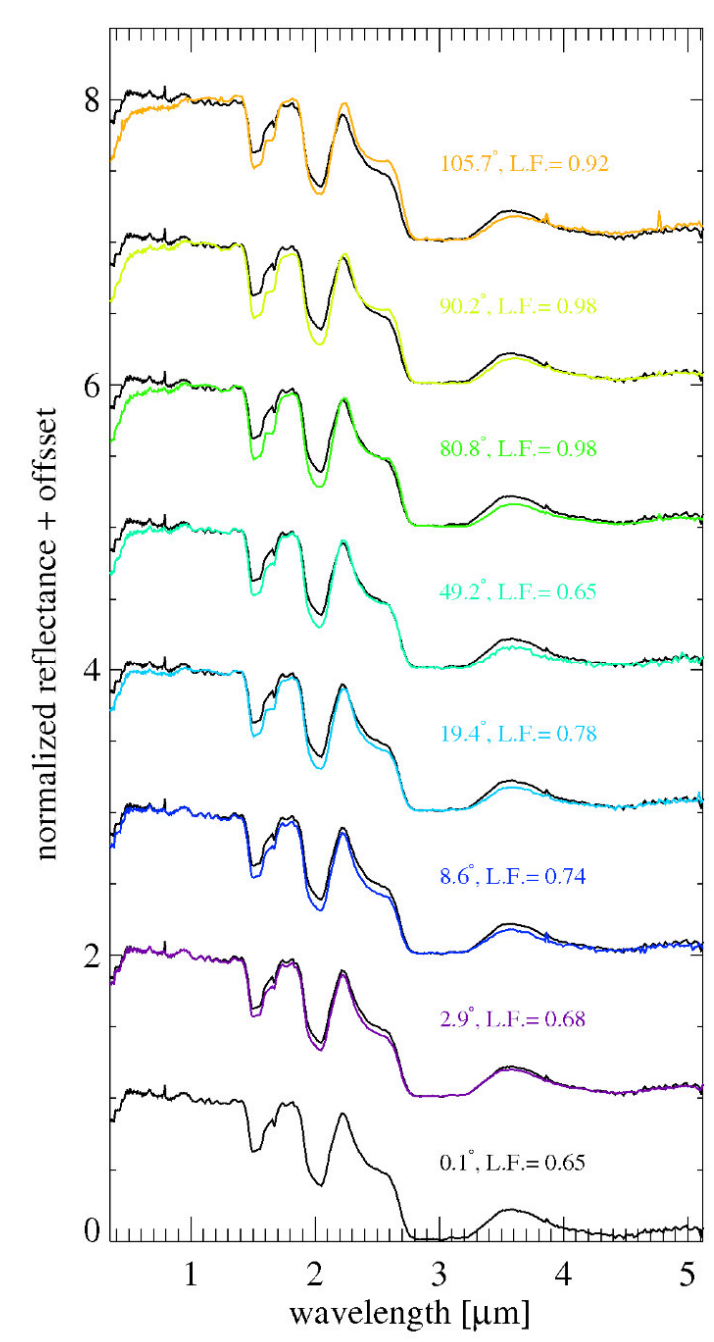

274

trend is reversed at $3.5 \mu \mathrm{m}$. This peculiar behavior can be partially explained by varying the relative contributions of single and multiple scattering at different wavelengths (related to different values of the single-scattering albedo $w$ as we will discuss later) and to a variation of single-particle phase

In Fig. 3 Rhea's normalized full-disk phase functions at various wavelengths are plotted. The coverage is fairly complete across the whole range, except for the $20^{\circ}-40^{\circ}$ region. The shape of the phase function is not constant with wavelength, which is obvious considering the dependence of the

279 spectrum on phase angle mentioned above. In particular, the OE width and reflectance at 


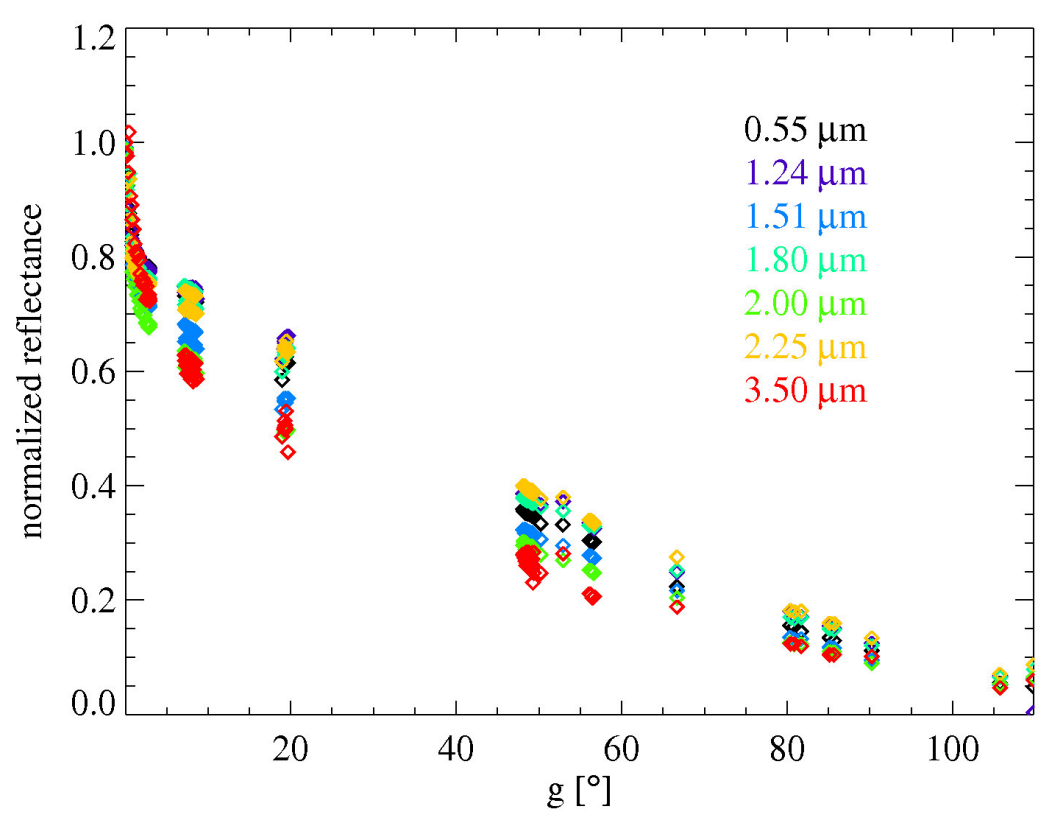

[FIGURE 3]

\section{Spectral fit}

The first step of our investigation is the interpretation of Rhea spectra in terms of physical characteristics of the surface involved in the scattering process. The principal properties that determine the observed spectral shapes are the composition (in this case water ice + contaminants) and the grain size. The presence of certain end-members is directly correlated with spectral signatures (absorption bands), while the grain size basically affects the depth of the bands as well as the IR slope (Clark and Lucey, 1984; Emery et al., 2005). In Hapke's model the spectral behavior is described by the single-scattering albedo $w$, whose value at each wavelength can be calculated once the end-members, their relative abundances, mixing mode and grain size distribution have been fixed. The single-scattering albedo cannot be directly compared to the observed spectra, because the 
reflectance at each wavelength depends on single scattering, which relies on the single-particle

296 phase function, and multiple scattering, which involves $w$ in a non linear way, as shown in (Eq.1).

297 Moreover, at low phase angle OE must be taken into account, while at high phase angles large scale surface roughness decrease the reflectance.

We have chosen to model Rhea's surface by means of a mixture of crystalline water ice and one organic contaminant. We used separately tholin from Khare et al. (1993), Triton tholin (McDonald et al., 1994; optical constants from Cruikshank, personal communication), Titan tholin (McDonald et al., 1994; Khare et al., 1984; optical constants from Cruikshank, personal communication) and hydrogenated amorphous carbon (ACH2) from Zubko et al. (1996). Optical constants for crystalline water ice are those derived by Warren (1984) $(0.35-1.25 \mu \mathrm{m}, 266.15 \mathrm{~K})$, Mastrapa et al. (2008)

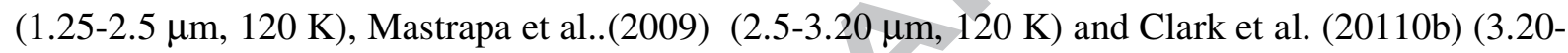
$5.12 \mu \mathrm{m}, 120 \mathrm{~K})$. We investigated areal mixing, intimate mixing and intraparticle mixing. In order to investigate spectral behavior of different mixtures without superimposing any grain size effect we studied only monodisperse particle diameter distribution (particles are all equal in size). This may lead to a non-unique solution, but as we shall see, provides information on the single particle phase function as a function of single particle albedo.

The other parameter fixed by the fitting procedure is the volume fraction of water ice $p$, with $p c=$ 1- $p$ being the amount of contaminants.

In order to retain a minimum number of parameters we decided to perform the spectral fit at high phase angle $\left(90^{\circ}\right)$ thus avoiding the $\mathrm{OE}$ surge. To remove the contribution of large scale surface roughness $K$ we fitted normalized spectra (normalization was performed at $1 \mu \mathrm{m}$ ). This choice also allows to minimize the geometrical effects of single-particle phase function which at this stage is assumed isotropic, but may further reduce the uniqueness of the solution.

\section{$\underline{4.1 \text { Optical constants }}$}


Summary plots of optical constants Vs wavelength used in this work are shown in Fig. 4-5. In the

VIS-NIR range optical constants from Warren et al. (1984) pertain to ice at $-7{ }^{\circ} \mathrm{C}$, whose temperature is too high if compared to Rhea's surface at $77 \mathrm{~K}$ (Pitman et al., 2010). However these values match reasonably well with the ones derived by Mastrapa et al. (2008) at $120 \mathrm{~K}$. Optical constants in the 3.2-5.1 $\mu \mathrm{m}$ range are from Clark et al. (2011b) and have been computed starting from Mastrapa's values at the same wavelengths. The temperature difference between Rhea's surface and ice for which optical constants are determined introduces a tolerable error in our calculations, because it only minimally affects the results concerning grain size and contamination. The organic compounds, listed above, used to contaminate water ice, all have the effect of producing a red spectrum towards the UV (Fig. 5). Tholin from Khare et al. (1993) have been produced by plasma irradiation in an iced 6:1 mixture of $\mathrm{H}_{2} \mathrm{O}$ and $\mathrm{C}_{2} \mathrm{H}_{6}$ at $77 \mathrm{~K}$. Titan and Triton tholin are instead obtained in gaseous phase by irradiation of 0.9:0.1 and 0.999:0.001 $\mathrm{N}_{2} \backslash \mathrm{CH}_{4}$ mixtures. ACH2 is obtained by arc discharge between carbon electrodes in $\mathrm{H}_{2}$ atmosphere.

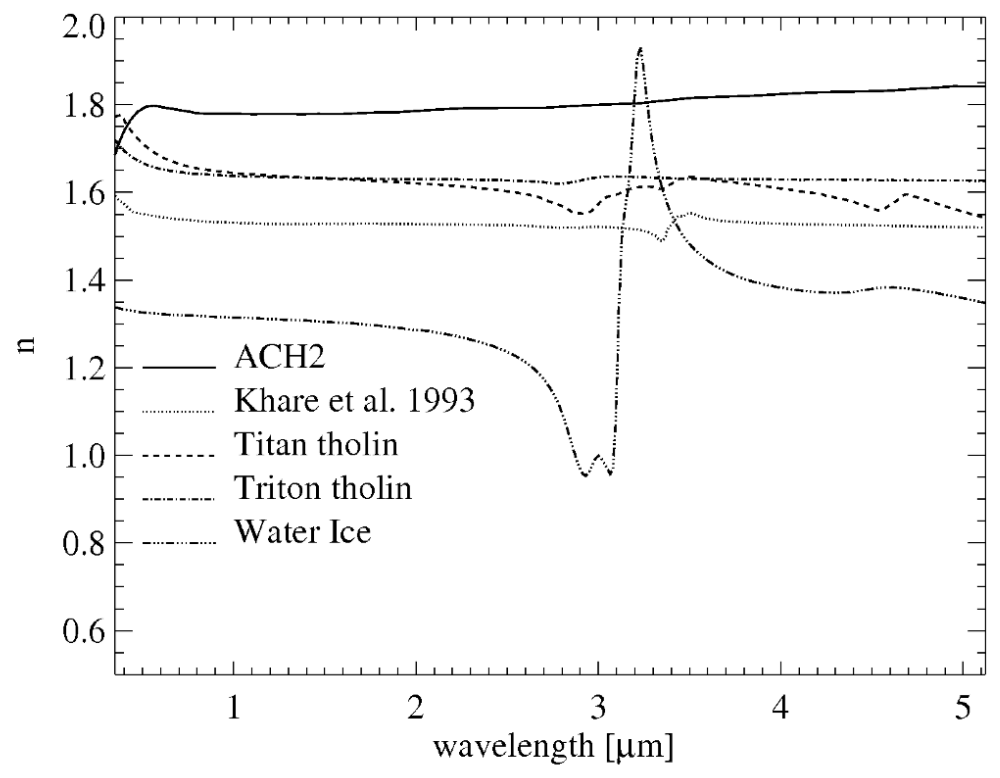

[FIGURE 4] 


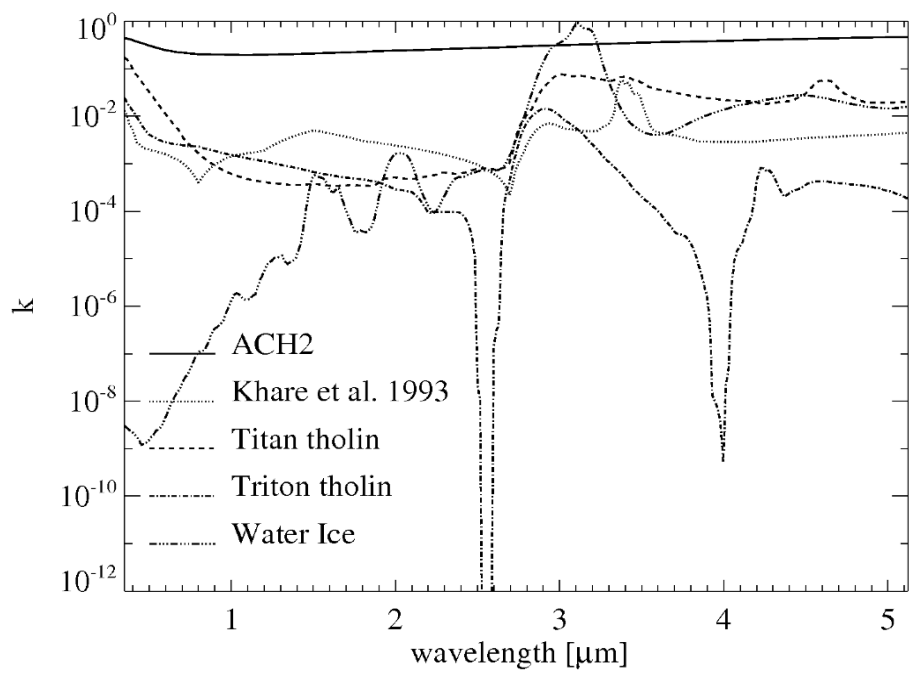

\subsection{Areal mixing}

We fitted observed spectra with different areal mixtures of two components where the main endmember is always water ice and the second is one among the selected organic contaminants. In areal mixing, the two different types of surface are characterized by different single-scattering albedo, and a beam of light interacts only with particles of the same composition. The resulting spectrum is a linearly-weighted average of the reflectances relative to the different regions. This kind of mixing is inefficient to produce the observed reddening towards UV. As an example we examine the cases of $\mathrm{ACH} 2$ and tholin (Khare et al., 1993). In the case of ACH2 the problem is mainly due to the fact that contaminant spectrum is not red enough to produce a sensible effect. It just reduces the reflectance across the whole spectrum without producing any absorption in the UV region, where water ice is strongly non-absorbing. In the normalized spectra this corresponds to an increment of reflectance of the darker wavelengths. This is shown in Fig. 6 (left panel) where the 
350

351

352

353

354

355

356

357

358

359

360

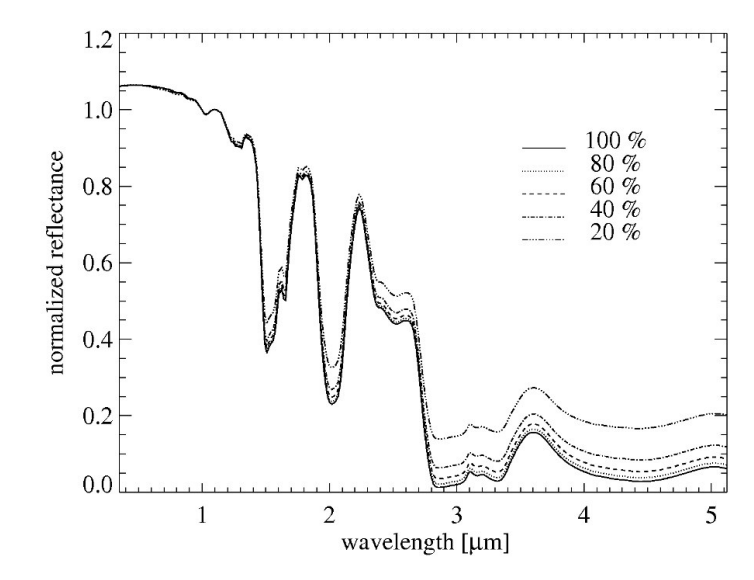

361

362 mixtures fits are summarized in Table 1.

\section{mixtures fits are summarized in Table 1.}

\section{FIGURE 6]}

results of five simulations with different abundances of $\mathrm{ACH} 2$ are plotted. The case of tholins (Fig. 6, right panel) is different because their spectra are not as flat as $\mathrm{ACH} 2$ far from UV region. In order to produce an effective feature towards short wavelengths many unobserved features are introduced in other regions of the spectrum. The outcome of this simulation is that the strong UV downturn observed in the Rhea spectrum it is not compatible with mixtures including compounds expected to be found on its surface. As an example, the best fit obtained with an areal mixture of water ice and Titan tholin is plotted in Fig. 7 (upper left panel). The results are similar using other types of contaminants. The fit, fairly good in IR, is completely lost in UV-VIS where a plateau is formed at the shortest wavelengths, missing the observed spectral downturn. Results for areal

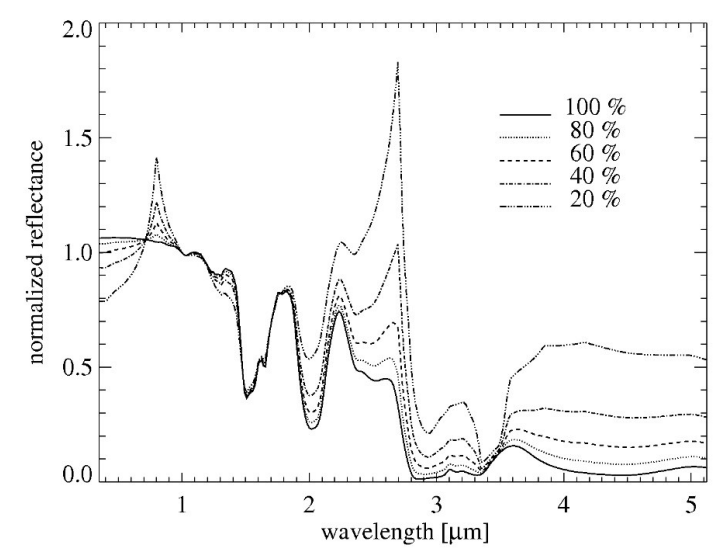



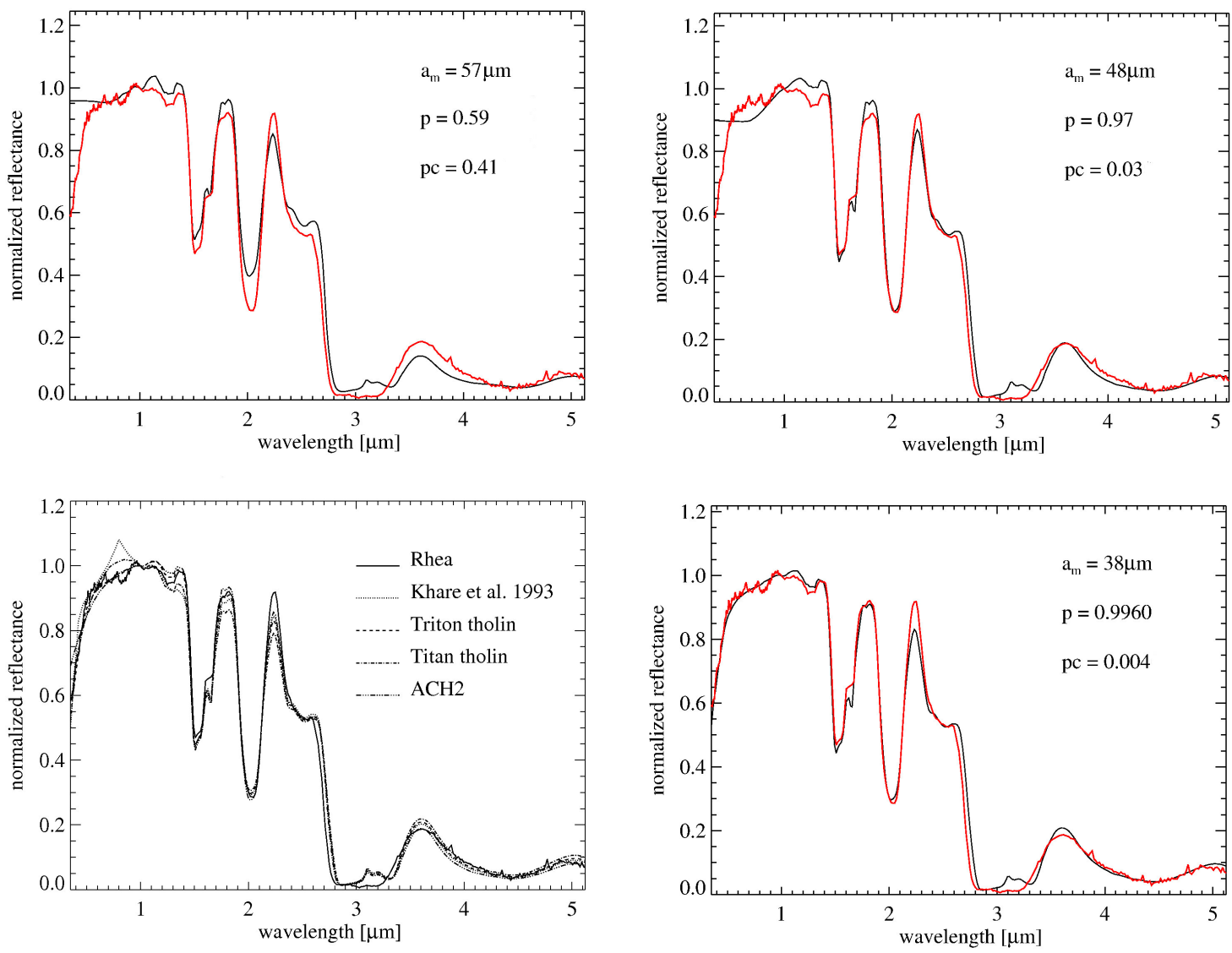

[FIGURE 7]

\subsection{Intimate mixing}

371 Similar to the case of areal mixtures we obtained fits to Rhea's spectrum considering intimate mixtures of water ice and the available contaminants. In an intimate mixture, particles of different

373 composition are in close contact (Clark, 1999), so this kind of mixing is also named "salt and 374 pepper" (Poulet et al., 2003). A single ray of light entering the medium in a given position interacts 375 both with water ice particles and contaminant particles. A small volume containing a statistically 376 significant number of particles behaves as if it had an effective single-scattering albedo given by the 
average of the albedo of the single particles. This kind of mixing is expected to be more efficient than areal mixing because at this stage spectral signatures (e.g. reddening) affect the effective single-scattering albedo and are stretched in higher order terms $\left(w^{2}, w^{3} \ldots\right)$ involved in the resulting reflectance, while in the case of areal mixtures, spectra of contaminants are only linearly combined. As a result, in intimate mixing, the darker component dominates the spectra signature (Clark, 1999). However, intimate mixing alone is still unable to reproduce the observed reddening of Rhea's spectrum for all the contaminants analyzed. Fig. 7 (upper right panel) shows the best fit obtained with an intimate mixture of water ice and Titan tholin. As with the result of the areal mixture the fit is acceptable in the IR but no reddening is produced towards the UV. The results of the fits are summarized in Table 2.

\section{[TABLE II]}

\section{$\underline{4.4 \text { Intraparticle mixing }}$}

In this approach we consider the surface covered by identical particles of water ice with small inclusion of contaminants. The single particle behaves as if it had effective optical constants derived from a combination, given by the Maxwell-Garnett equation, of optical constants of ice and inclusions. For small amount of contaminants (which is the case of this work) the Maxwell-Garnett equation gives results similar to those obtained by a weighted average of the optical constants (Cuzzi and Estrada, 1998). This kind of mixing is the most efficient means of producing reddening because it exaggerates spectral differences, working directly with the complex refractive indices. As shown in Fig. 7 (bottom left panel), intraparticle mixing produces the required UV reddening with every contaminant considered in this work, although the type of reddening change from one contaminant to another. Extremely low concentrations of $\mathrm{ACH} 2(0.01 \%)$ are able to introduce UV 
401 reddening, but the resulting spectrum falls too quickly towards short wavelengths before $1 \mu \mathrm{m}$ and

402 it is not steep enough below $0.4 \mu \mathrm{m}$. Titan tholin produces good fits at the shortest wavelengths but 403 it is not sufficiently absorbing towards $1 \mu \mathrm{m}$. Tholin from Khare et al. (1993) shows a good 404 agreement below $0.5 \mu \mathrm{m}$ but has an unobserved feature at $0.8 \mu \mathrm{m}$. The best fit (Fig. 7, bottom right 405 panel) is obtained with Triton tholin which reproduces even the change of slope in the spectrum 406 around $0.5 \mu \mathrm{m}$. The particle size varies depending on the chosen contaminant. However, if we 407 discard the results given by Titan tholin which produces the worst fit in the IR where the spectrum 408 is more sensitive to grain size, we find that the particles diameter is limited to the range $40-50 \mu \mathrm{m}$.

409 This diameter can be considered as an average size of particles, once we assume a monodisperse 410 grain size distribution. Some discrepancies between the final fit and observed spectrum due to 411 particle size are discussed in the next section where the best spectral fit is shown. Results for 412 intraparticle mixtures fits are summarized in Table 3.

\section{3 [TABLE III]}

\section{$414 \quad \underline{4.5 \text { Best spectral fit }}$}

415 Considering the results presented in previous sections the best way to reproduce the spectral 416 properties of Rhea is to assume an intraparticle mixture of $99.60 \pm 0.05 \%$ water ice and $0.40 \pm 0.05 \%$ 417 Triton tholin, with a grain diameter of 38.0 $0.5 \mu \mathrm{m}$ (Fig. 7, bottom right panel). Uncertainty on the 418 derived values is related to the procedure we applied to perform the fit, as explained in Appendix 419 A. Despite the small number of free parameters and end-members, the simulated spectrum fits well 420 the observed spectrum. With such a low amount of tholin as a contaminant in the ice, other tholin 421 absorption bands have a small effect on the infrared spectrum where ice is more absorbing. VIS reddening as well as water ice bands are very well reproduced. The secondary absorption band of crystalline water ice at $1.65 \mu \mathrm{m}$, which is shown in simulated spectrum, cannot be confirmed in

424 VIMS data because in the wavelength range $1.60-1.66 \mu \mathrm{m}$ the measured signal is affected by the 425 presence of an order sorting filter on the detector. Consequently, the measured signal in that region 
426 is replaced by an interpolated value. One of the stronger discrepancies is in the peak at $2.2 \mu \mathrm{m}$. This

427 problem is shown in all the mixtures that have been analyzed so does not depend on the spectral

428 properties of the contaminant but is instead most probably due to the chosen grain size distribution.

429 In the case of monodisperse grain size distribution all the particles are equal and the contribution

430 from smaller particles (with particle size similar or smaller than the wavelength), which certainly

431 are present in a real distribution of sizes, is not considered. It must be noted that Hapke's model is

432 developed in the geometric optics domain, so normally does not deal with grain size smaller than

433 the wavelength. Clark et al. (2011b) have extended the Hapke model to include the diffractive

434 scattering and absorption effects from sub-micron particles. Adopting a distribution of sizes which

435 includes smaller particles it might be possible to reproduce the peak at $2.2 \mu \mathrm{m}$. Another part of the

436 spectrum where the fit is lost for all the mixtures we deal with, is given by the absorption band at 3

$437 \mu \mathrm{m}$. In simulated spectra the $3.1 \mu \mathrm{m}$ Fresnel peak is always visible, while in the measured spectra it

438 completely disappears. The absence of the Fresnel peak cannot be completely attributed to the

439 relative abundance of amorphous versus crystalline ice, as in amorphous ice the Fresnel peak

440 doesn't fully disappear as shown by (Mastrapa et al., 2009). Moreover ground-based telescopic

441 spectra of Rhea also attest to the crystalline nature of $\mathrm{H}_{2} \mathrm{O}$ dominating its surface (Cruikshank et al.

442 2005; Emery et al. 2005). The absence of the Fresnel peak in Rhea's spectra (as in the case for the

443 others icy bodies of the saturnian system) is probably due again to a grain size effect. In large

444 particles light at $3 \mu \mathrm{m}$ is almost completely absorbed, given the high value of $k$ and the longer path

445 that light travels inside the grain. This implies that when we deal with big grains the light scattered

446 by the particles is the one coming from surface reflections (that involves $n$ ) and which generates the

447 Fresnel peak. Scattered light from smaller particles is the result of both surface reflection and

448 internal reflection, thus the Fresnel peak is minimized. Since in our analysis the grain size is around

$44940 \mu \mathrm{m}$ and the contribution from small particles is neglected, the resulting spectrum exhibits an

450 evident peak at $3.1 \mu \mathrm{m}$. 
451 An intraparticle mixture of water ice and Triton tholin was adopted in Cruikshank et al. (2005)

452 where a fit of Rhea spectrum was performed applying the Shkuratov model. It is interesting to note

453 that both approaches require a comparable amount of embedded contaminant $(0.4 \%$ in this work,

$4540.2 \%$ in Cruikshank et al. (2005)) and they both reproduces the reddening in the UV, even if the 455 adopted spectral models and the surface modeling are different. It reinforces the idea that 456 intraparticle mixing is the best approach to explain the UV feature and that Triton tholin is a 457 reasonable candidate as the water ice contaminant.

\section{Phase function fit}

Results from the spectral fit allow us to determine the mixture (type of contaminant and mixing modality) and the grain size. The knowledge of these two parameters enables us to compute singlescattering albedo $w$ which determines the spectral behavior of the observed surface. This quantity is involved in Eq. 1, which in our approach depends on 5 parameters (apart from $w$ ). The possibility to fix the single-scattering albedo reduces the complexity of phase function fit procedure and allows the decoupling of spectral effect from photometric ones.

A phase function fit at each wavelength available in VIMS range has been performed, using the surface model given by the best spectral fit that is represented by an intraparticle mixture of water ice and Triton tholin $(99.6 \%-0.04 \%)$, with $38 \mu \mathrm{m}$ grain size. Parameters relative to the $\mathrm{OE}\left(B_{0}, \mathrm{~h}\right)$ are allowed to vary along the spectrum because, as we mention in section 2 , we want to take into account the $\mathrm{CBOE}$ as well which can depend on wavelength. Even the single-particle phase function $(b, v)$ parameters can vary with wavelength because the single-scattering albedo has spectral variation and modifies the scattering properties of the grain. The large-scale surface roughness parameter $(\bar{\theta})$ is constant all over the spectrum because it accounts only for geometric effects due to surface structures. Details on the fit procedure are in Appendix. In Table 4 the best phase function fit variables for each VIMS channel are reported. 
475 As we deal with particles that are in average larger than the wavelength, we do not expect a direct

476 correlation between the values of parameters obtained by the fit and the wavelength itself. On the

477 other hand, the single-scattering albedo, which determines the contribution of scattering in the light

478 extinction process, plays a fundamental role for the photometric properties at a given wavelength.

479 This implies that the estimated parameters should be correlated with the single-scattering albedo

480 rather than with the wavelength.

\section{1 [TABLE IV]}

482

\section{$\underline{5.1 \text { Residuals }}$}

483

484

$485 \quad r e s=\Sigma\left(\frac{r_{i}^{m}-r_{i}^{c}}{r_{i}^{m}}\right)^{2}$, formula:
Fits are performed minimizing the residuals that are calculated for each wavelength following the

where $\mathrm{r}_{\mathrm{i}}^{\mathrm{m}}$ is the measured absolute reflectance at $i$ 'th phase angle while $\mathrm{r}_{\mathrm{i}}^{\mathrm{c}}$ is the value computed by the fit procedure (solar spectrum used to calibrate VIMS data is derived from Thekaekara (1973)). This kind of choice aims to give equal weight to the head and the tail of the full-disk phase function. Reconstructed full-disk phase functions for those wavelengths where the albedo is very low are often dominated by noise, and the corresponding fit parameters values are thought to have no physical meaning. Additionally, they produce high residuals because the model is unable to perform a satisfactory fit. For this reason we have considered for the following analysis only wavelengths with well determined full disk phase function and low value of the residuals. We found that for a residual value of 1 the full disk phase function can be considered well reproduced. From this point to the end of the paper we refer only to results relative to residuals lower than 1 , unless explicitly written. 
497

498

499

500

501

502

503

504

505

506

In Fig. 8 (upper left panel) fit residuals for each band are plotted against the value of singlescattering albedo computed for the correspondent wavelength. As we can see the accuracy of the fits increases with larger values of the single-scattering albedo. This effect is due to two reasons. The first is that for those wavelengths corresponding to a higher value of $w$ the signal to noise ratio is typically higher and reconstructed phase functions are more accurate. The second is that at low values of the single-scattering albedo the dominating process is single scattering. In this regime the full-disk phase function is more sensitive to single-particle phase function and worse regression accuracy indicates that the Heyney-Greenstein expression is not able to completely describe the scattering process.
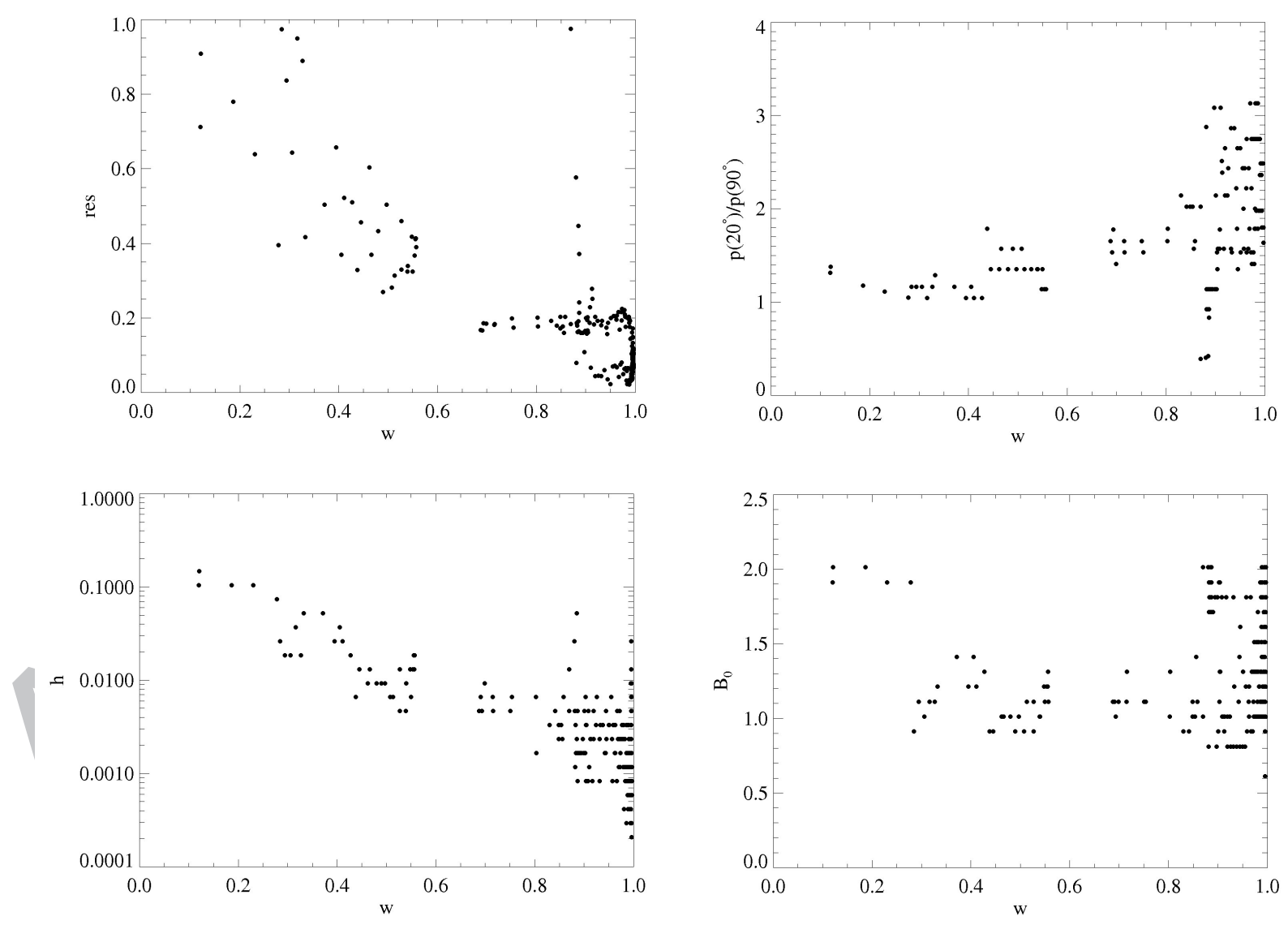

507

508

FIGURE 8] 


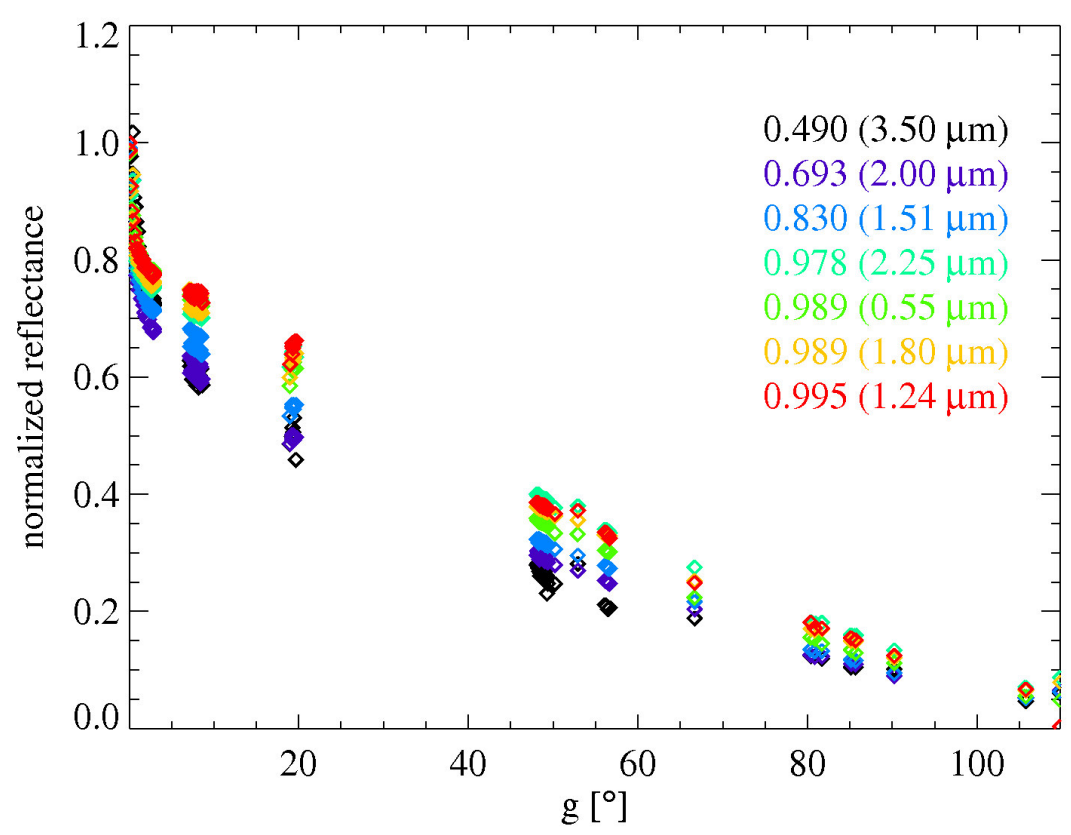

509

[FIGURE 9]

$512 \quad 5.2$ Single-particle phase function

513 In fig. 10 the distributions of $b$ and $v$ parameters are plotted. In these graphs it is shown how many

514 times a certain value of the parameter occurs in a given range of $w$ values. According to McGuire

515 and Hapke (1995) a clear spherical particle should have $\mathrm{b} \sim 0.5-0.7$ and $\mathrm{v} \sim-0.9$. Most of our $b$

516 values are slightly lower (0.3-0.4) as it's expected for real grains that are not perfectly spherical.

517 Moreover $b$ values should decrease with increasing absorption and it is what we found, since the

518 fitted values shows a positive correlation with $w$. Concerning the $v$ values, they are negative for a

519 large part but greater than -0.9 (average value is -0.5 ), again as expected for not perfectly spherical 520 particles. 

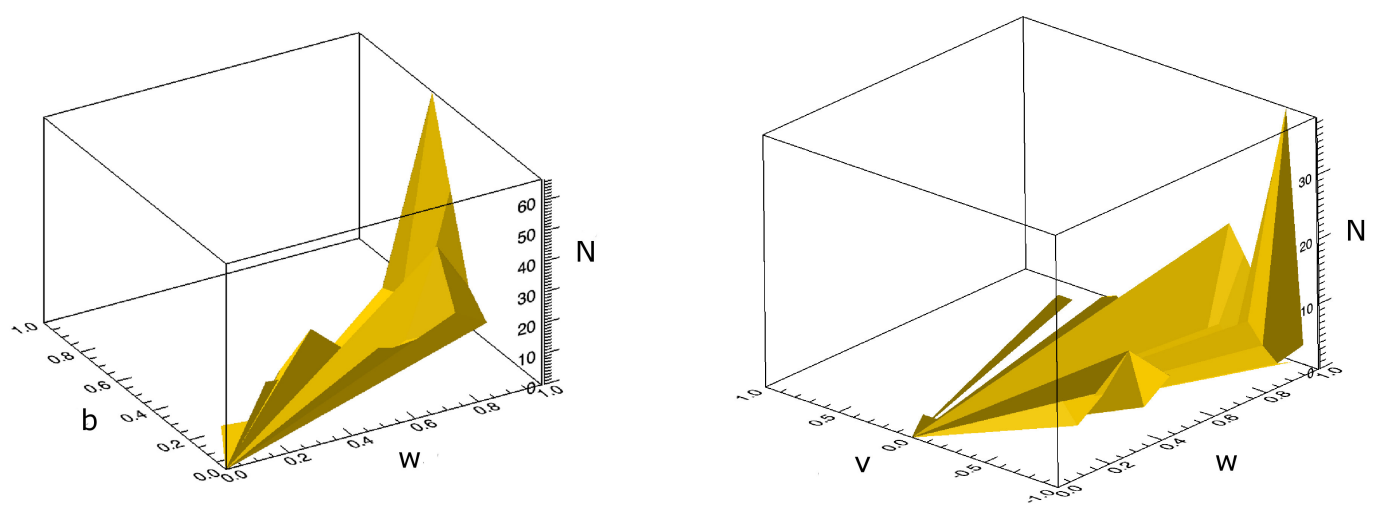

\section{[FIGURE 10]}

In order to show any relation between single-particle phase function and single-scattering albedo is useful to investigate the final shape of $p(g)$. In Fig. 8 (upper right panel), the ratio $p\left(20^{\circ}\right) / p\left(90^{\circ}\right)$ vs $w$ is shown. This choice aims to point out the trend of the single-particle phase function at intermediate phase angles, avoiding the phase angle regions where the contribution of the OE and large scale roughness become relevant. The $p\left(20^{\circ}\right) / p\left(90^{\circ}\right)$ ratio increases with single-scattering albedo meaning that reflectance increases at intermediate phase angles $\left(10^{\circ}-60^{\circ}\right)$ for more transparent particles. This behavior can be explained by the possibility for a light ray to undergo multiple internal reflections in a non-absorbing particle, producing an higher backscattering lobe respect to a darker particle, where surface reflection dominates and produces a quite flat singleparticle phase function at phases $<90^{\circ}$ (Hapke, 1993, p. 77, Fig. 5.7a). This result is better shown in 
538

539

540

541

542

543

544

545

546

547

548

549

550

551

552

553

554

555

556

557

558

559

560

561

Fig. 9, where normalized full disk phase functions are plotted ordered according to increasing values of single-scattering albedo (listed along with their relative wavelengths).

\subsection{Opposition effect}

As anticipated in previous sections we choose to model the opposition effect following the results developed in Hapke (1993) which consider only shadow hiding (SHOE). This model depends on two parameters $\left(B_{0}, h\right)$ which describes respectively the amplitude and angular width. Nonetheless, another mechanisms, coherent backscattering opposition effect (CBOE), has been recognized as contributing to the OE (Hapke et al., 1998; MacKintosh and Sajeev, 1998; Shkuratov et al. 1999b; Hapke, 2002). Given these reasons the interpretation of results concerning the OE is not trivial, because the two parameters $\left(B_{0}, h\right)$ must describe the contributes of both SHOE and CBOE. The two effects have different characteristics that help us discern between them. The major difference is that angular width of SHOE doesn't depend on wavelength, while the CBOE does (MacKintosh and Sajeev 1998; Hapke 2002). The second one is that the SHOE width extends up to $10^{\circ}$ or more while the CBOE is limited to a width of $2^{\circ}-3^{\circ}$ at most (Hapke et al. 1998, Shkuratov et al. 1999b). Additionally we must consider that SHOE is a single-scattering effect while the CBOE develops in a multiple-scattering process. Therefore, we expect that the SHOE dominates for wavelengths corresponding to low values of $w$ while the CBOE dominates at wavelengths where $w$ values is close to 1 .

Figure 8 (bottom left panel) shows the plot $h$ against $w$. The values of $h$ spread over almost three orders of magnitude (0.0002-0.14) giving an OE half width $\Delta g \approx 2 h$ ranging between $0.01^{\circ}-16^{\circ}$, and show a clear correlation with single-scattering albedo. At low values of $w$ we have high $h$ while the opposite is true when the albedo is low. This behavior reveals the presence of two competing 
562

563

564

565

566

567

568

569

570

571

572

573

574

575

576

577

578

579

mechanisms in the OE: the CBOE for wavelengths with high value of albedo and the SHOE for the opposite case. This fact agrees with the argument that the CBOE depends on multiple scattering and the SHOE on single scattering. Moreover, we would expect that the $h$ values at low $w$ would be nearly constants, since SH is independent of wavelength. What we see, in fact, is that spreading reduces considerably towards small values of $w$ with $h$ approaching 0.1 .

In the $\mathrm{SH}$ regime a rough estimation of the porosity of water ice particles on the surface can be computed from the following relation (Hapke 1993):

$h \approx-\frac{3}{8} \ln (1-\phi)$

where $\phi$ is the filling factor. The value of $h$ we chose to use is 0.1 , which is referred to low values of the single-scattering albedo, where single scattering dominate and the $\mathrm{OE}$ is due to $\mathrm{SH}$. Moreover this value is almost constant with $w$, so it is independent of wavelength, which is what we expect for the SHOE. With this choice we obtain $\phi=0.23$. However, considering that a real grain size distribution has a non null dispersion and that the particle diameter we have measured is just an average value the filling factor can be higher. Assuming a grain size distribution of the form:

we obtain $\Phi=0.35$. The derived porosity is then in the range $65-77 \%$. These values are lower than those derived in a similar study by Domingue et al. (1995). It must be noted that in Domingue's work the analysis was performed at 0.47 and $0.55 \mu \mathrm{m}$, where the single-scattering albedo is close to 1 and the CBOE contribution is important, reducing $h$ and increasing the estimated porosity.

Regarding the amplitude of the opposition effect $B_{0}$ (Fig. 8, bottom right panel) we do not find any particular trend with $w$, apart from the fact that values have a larger spreading for single-scattering 
583

584

585

586

587

588

589

590

591

592

593

594

595

596

597

598

599

600

601

602

603

604

605

albedo close to 1 tending to be higher respect to the rest of the spectrum. This could be an indication of the superimposition of the $\mathrm{CB}$ on $\mathrm{SH}$, but a compensating effect due to the unsatisfactory modeling of the single-particle phase function (backscattering region) cannot be excluded.

\section{$\underline{5.4 \text { Large-scale surface roughness }}$}

The large-scale surface roughness is characterized by the $\bar{\theta}$ parameter which Hapke (Hapke, 1993) interprets an average slope of the surface structures. This is the only parameter that doesn't depend on wavelength in our fit procedure. To constrain it we performed fits of full-disk phase functions for each value of $\bar{\theta}$ in the range $10^{\circ}-35^{\circ}$ and chose the one that produced the smallest residual. We found a final value of $\bar{\theta}=33^{\circ}$ (we must point out that all the values above $30^{\circ}$ gave similar fits). The result obtained seems too high if related to common slopes of craters and other topographic structures, and exceeds the values found by Domingue et al. (1995), while is close to the one found by Buratti (1985) for Mimas. Recent experimental analysis performed by Shepard and Helfenstein (2007), has shown that the value of $\bar{\theta}$ is not only affected by subpixel topography but also, and mainly, by roughness on the scale of particles clumping which can produce fairly high slopes, related to the angle of repose of the regolith, and consistent with our determination.

Typically, in phase function fit procedures, the estimated values of single-scattering albedo and surface roughness show a certain degree of correlation, which of course has no physical meaning and reveals a degeneration in the inversion process.

In our approach the evaluation of $w$ comes from the spectral fit and it's completely independent on the determination of the roughness parameter, so the degree of degeneration of the regression is 
606

607

608 609

reduced making the result more reliable. However, it must be noted, as pointed out in Davidsson et al. (2009), that the treatment of surface roughness in Hapke's theory relies on the assumption of "small mean slope" $\left(\bar{\theta}<10^{\circ}\right)$, which allows to obtain analytical solutions. The value we retrieved from the phase function analysis is far beyond this limit and must be considered with care.

Another issue is represented by the fact that in Hapke's theory multiple scattering between the facets composing the surface is neglected which is not applicable for high albedo materials and rough surfaces. In particular, icy surfaces have high albedo and, as shown in Shepard and Helfenstein (2007), the sub-centimeters scale roughness implies fairly large slope angles (typically above $\left.10^{\circ}\right)$.

This would limit the applicability of the roughness correction only to low albedo media, which is not the case of icy surface, unless the analysis is restricted to wavelengths where strong absorption bands are located (eg. 2.0 and $3.0 \mu \mathrm{m}$ ). In our analysis the derived $\bar{\theta}$ value produces good fits both for high and low reflectance values (e.g. 1 and $2 \mu \mathrm{m}$ ). This would imply that the effect of multiple scattering is less relevant than the "small mean slope" approximation.

\section{Feedback on the spectral fit}

The results of the phase function fits can be used to improve the spectral fit, which represented the starting point of our analysis. The spectral fit, as explained in section 4.0, was performed assuming an isotropic phase function and at phase angles large enough to avoid contributions from any OE. Now, for each phase angle it is possible to compute the absolute spectrum, removing the approximation of an isotropic single-particle phase function, including the $\mathrm{OE}$ and surface roughness. We have recalculated the spectrum at $g=90^{\circ}$, the one chosen to perform the starting spectral fit, and plotted it in Fig. 11. The agreement between the fitted spectrum and the measured one is almost perfect throughout the VIS-NIR region. Similar fits have been performed for each 
629 spectrum (phase angle) of the dataset. As example the results relative to spectra at $g=2.1^{\circ}$ and $630 g=49.4^{\circ}$ are plotted in Fig. 12.

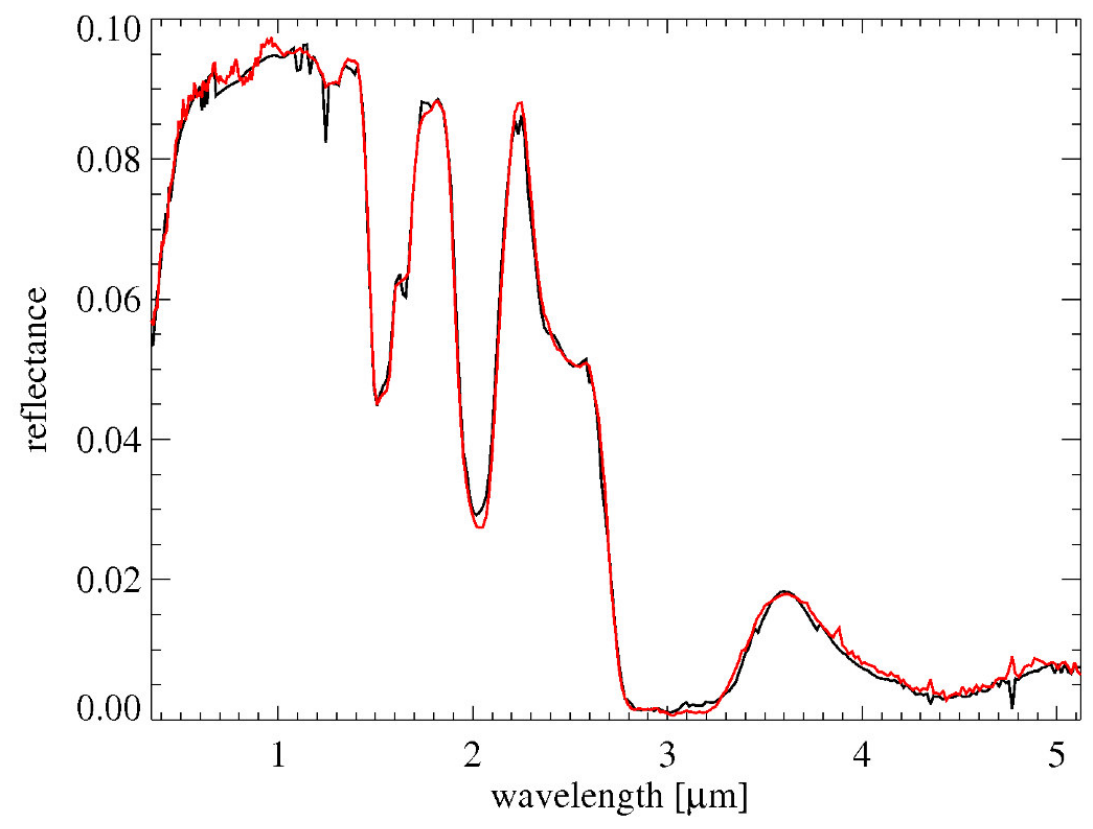

631

\section{[FIGURE 11]}
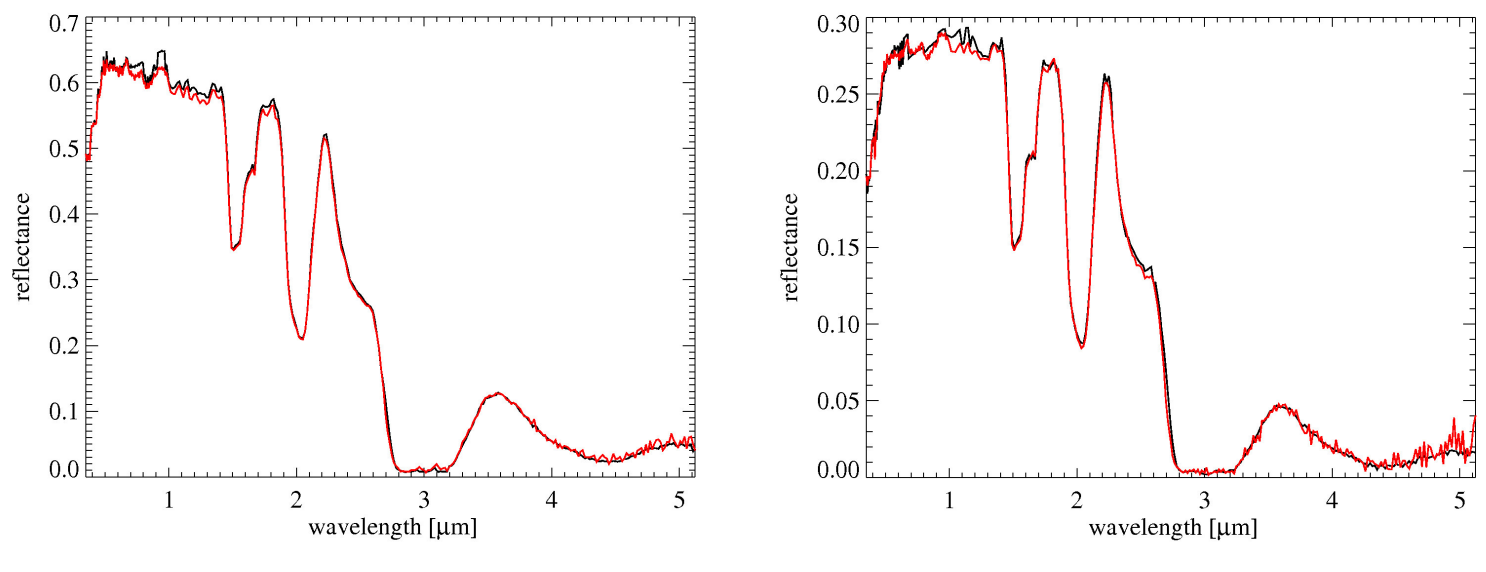
This high level of fit accuracy is possible because we deal with a large number of free parameters and this allows us to tune the reflectance at each wavelength. This represents also the limitation of this approach. In fact, any mismatches in the spectral fit due to grain size effects, as shown in section 4.5 for the reflectances at $2.2 \mu \mathrm{m}$ and $3.1 \mu \mathrm{m}$, or due to an incomplete knowledge of the ice optical constants, for instance, can be compensated by the contribution of the single-particle phase function. The spectral fit will be optimal but we shall be misled in the interpretation of the scattering mechanisms at work.

On the other hand, the trends observed in Fig. 8, 9 and 10 between phase curve parameters and the single-scattering albedo points out some degree of physical correlation among those variables. For instance, it is not totally unexpected to find that brighter surfaces have a narrower width of the opposition effect than darker surfaces; the contribution of multiple scattering within the grains attenuates the shadow hiding effect limiting it to a narrow region around the zero phase condition. Also, for planetary surfaces, the slope of the linear part is larger for brighter surfaces and this is consistent with the results shown in Fig. 8-9.

In other words, we could use these correlations to give preliminary estimates of the phase curve parameters to be used in the determination of the spectral fit; this approach can prove valuable also in case of incomplete or undersampled phase curves.

\section{Summary and conclusions}

We have studied surface spectrophotometric properties of Rhea, the largest icy satellite in the saturnian system. The analysis has been performed on spectra acquired by VIMS onboard Cassini, in the $0.35-5.12 \mu \mathrm{m}$ spectral range, covering the $0.08^{\circ}-110^{\circ}$ phase angle interval. This approach enabled us to investigate the compositional state of the ice covering the moon, the agglomeration state of the medium and the roughness properties. Hapke's spectral and photometric model has been 
661 used for the analysis. Four different organic compounds have been investigated as water ice 662 contaminants in order to model the reddening observed towards the UV. The best spectral fit in our 663 model is represented by an intraparticle mixture of crystalline water ice $(99.6 \%)$ and Triton tholin $664(0.4 \%)$, with a grain size (diameter) of $38 \mu \mathrm{m}$. Major discrepancies between the measured and fitted spectrum can be addressed to a grain size effect, because our particle diameter distribution represents only the average size of the particles and therefore neglects the contribution of grains with dimensions comparable to or less than the wavelength.

Once the composition of the particles in the model was frozen it was possible to investigate the photometric properties of the satellite fitting the full-disk phase function at each wavelength. The picture that emerges is that the dominating parameter in the scattering process is the singlescattering albedo $w$, showing correlation with the single-particle phase function and OE. No particular dependence of $\mathrm{OE}$ on wavelength was found, as expected, considering that the typical grain size is larger than $\lambda$. The analysis of the $\mathrm{OE}$ indicates that both $\mathrm{SH}$ and CB are active, and their relative contribution depend on the single scattering albedo value. Measuring the $\mathrm{OE}$ angular width $h$ in the SH regime we estimated a porosity varying between $65-77 \%$.

The relatively high value of the roughness parameter $\left(\bar{\theta}=33^{\circ}\right)$ is not compatible with the mean slope of surface structures like craters, depressions or other reliefs. This points to a correlation of $\bar{\theta}$ with roughness on smaller scale, possibly on the order of centimeters, confirming the Shepard and Helfenstein (2007) results, and providing an estimation of the regolith angle of repose.

The next step will be to apply the methodology described in this paper to the other full disk observations of the Saturn's icy satellites. From this point of view the huge VIMS dataset represents an extremely useful resource, given the large number of observations over a wide variety of geometric conditions. Our goal will be to determine the distribution of contaminants in the saturnian 
685

686

687

688

689

690

691

692

693

694

695

696

697

698

699

700

701

702

703

704

705

706

useful tool for characterizing the effect of processes shaping the surfaces of these bodies: interactions with other moons, with the rings, and with the saturnian magnetosphere, as well as the surface activity of the moon itself.

As a future development we intend to investigate different kinds of grain size distributions involving even smaller particles (e.g. as done by Clark et al., 2011b). This new approach is beyond the limit of geometric optics applied in the present formulation of Hapke's model and requires to use Mie theory (Mie, 1908) to compute single-scattering albedo of the particles.

\section{$\underline{\text { Acknowledgments }}$}

The authors M.C., F.C., G.F., P.C., A.C. and F.T. acknowledge support from an Italian Space Agency grant.The authors wish to thank the referees for their constructive comments.

\section{APPENDIX: FIT PROCEDURE}

Performing an inversion of a model with several free parameters is a challenging task. The most common problem is to discriminate between different solutions that give similar results. Fitting algorithms are able to find minima in the parameters space but it is difficult to discriminate between local and absolute ones. To overcome this problem we adopted a very simple and transparent method. We determined a grid in the parameter space through a quantization of the parameters over the full range of variability. For each point of the grid (a single combination of the parameters) we calculated the model prediction and compared it to the data. The best prediction represents the final 
707

708

709

710

711

712

713

714

715

716

717

718

719

720

721

722

723

724

725 results of the fit. This method correctly finds the absolute minimum if the parameters space is sufficiently sampled. The quantization we chose is related to the degree of precision needed by the fit. At the same time the variability range for unbounded parameters has been fixed considering a range of values with physical sense.

Concerning the spectral fit we chose a quantization for the particle diameter $a_{m}$ of $1 \mu \mathrm{m}$ in a range extending from 10 to $100 \mu \mathrm{m}$; we knew from previous analysis that higher values where unnecessary and that diameter values lower than $10 \mu \mathrm{m}$ would have broken the limits given by geometric optics in Hapke model. The water ice mixing percentage range changes corresponding to the different mixtures, and in any case $p$ was 51 . The quantization "step" of the parameters has been chosen as the minimum variation that could create an appreciable change in the output, consequently the fitted value can be assumed with an uncertainty of half "step".

For the phase function fit the various parameters have been quantized in the following way:

$\mathrm{B}_{0} \in[0 ; 2], \mathrm{B}_{0 \mathrm{i}}=\mathrm{i} \cdot 0.1, \mathrm{i}=0,1,2, \ldots, 10$

$h \in[0.0001 ; 0.1], h_{i}=10^{(-4+0.15 \cdot i)}, i=0,1,2, \ldots, 30$

$b \in[0 ; 9], b_{i}=i \cdot 0.1, i=0,1,2, \ldots, 9$

$\mathrm{v} \in[-1 ; 1], \mathrm{v}_{\mathrm{i}}=-1+\mathrm{i} \cdot 0.1, \mathrm{i}=0,1,2, \ldots, 20$

$\bar{\theta} \in\left[10^{\circ} ; 35^{\circ}\right], \bar{\theta}_{\mathrm{i}}=\mathrm{i}, \mathrm{i}=10^{\circ}, 11^{\circ}, 12^{\circ}, \ldots, 35^{\circ}$ 
Bibliography

727

728

729

730

731

732

733

734

735

736

737

738

739

740

741

742

743

744

745

746

747

748

749

750

751

752

753

754

755

756

757

758

759

760

761

762

763

Bowell, E., Hapke, B., Domingue, D., Lumme, K., Peltoniemi, J., Harris, A.W., 1989. Application of photometric models to asteroid. In: Binzel, R.P., Gehrels, T. and Matthews, M.S., (Eds.), Asteroids II, University of Arizona Press, Tucson, pp. 524-556.

Brown, R.H., Baines, K.H., Bellucci, G., Bibring, J.-P., Buratti, B.J., Capaccioni, F., Cerroni, P., Clark, R.N., Coradini, A., Cruikshank, D.P., Drossart, P., Formisano, V., Jaumann, R., Langevin, Y., Matson, D.L., McCord, T.B., Mennella, V., Miller, E., Nelson, R.M., Nicholson, P.D., Sicardy, B., Sotin, C., 2004. The Cassini Visual and Infrared Mapping Spectrometer (VIMS) investigation. Space Sci. Rev. 115 (1-4), 111168.

Buratti, B.J., 1985. Application of a radiative transfer model to bright icy satellites. Icarus 61, 208-217.

Buratti, B.J., Hicks, M.D., 2003. The Dark side of Iapetus: A model that finally works? Bull. Am. Astron. Soc. 35, 915 (abstract).

Buratti, B.J., Mosher, J.A., Nicholson, P.D., McGhee, C.A., French, R.G., 1998. Near-infrared photometry of the saturnian satellites during ring plane crossing. Icarus 136, 223-231.

Buratti, B.J., Hicks, M.D., Soderblom, L.A., Britt, D., Oberst, J., Hillier, J.K., 2004. Deep space photometry of the nucleus of Comet 19P/Borrelly. Icarus 167, 16-29.

Ciarniello, M., Capaccioni, F., Filacchione, G., Coradini, A., Cerroni, P., Tosi, F., 2010a. VIS-IR spectral modeling of Rhea and Enceladus. EGU General Assembly 2010, 6177 (abstract).

Ciarniello, M., Capaccioni, F., Filacchione, G., Coradini, A., Cerroni, P., Tosi, F., Stephan, K., 2010b. Spectrophotometric analysis of Rhea surface scattering properties. Lunar Planet. Sci. XLI, 1643 (abstract).

Clark, R.N., Lucey, P.G., 1984. Spectral properties of ice-particulate mixtures and implications for remote sensing. I - Intimate mixtures. J. Geophys. Res.89, 6341-6348.

Clark, R.N., Owensby, P.D., 1981. The infrared spectrum of Rhea. Icarus 46, 354-360.

Clark, R.N., 1999, Chapter 1: Spectroscopy of Rocks and Minerals and Principles of Spectroscopy, Manual of Remote Sensing, (A.N. Rencz, ed.) John Wiley and Sons, New York, p 3-58, 1999.

http://speclab.cr.usgs.gov/PAPERS.refl-mrs/refl4.html

Clark, R.N., Brown, R.H., Jaumann, R., Cruikshank, D.P., Buratti, B., Baines, K.H., Nelson, R.M., Nicholson, P.D., Moore, J.M., Curchin, J., Hoefen, T., and Stephan, K., 2008, Compositional mapping of Saturn's satellite Dione with Cassini VIMS and implications of dark material in the Saturn system, Icarus, 193, 372-386.

Clark, R. N., R. Carlson, W. Grundy, and K. Noll, 2011a, Observed Ices in the Solar System. In: Solar System Ices, Murthy Gudipati, ed., in press.

Clark, R. N., D. P. Cruikshank, R. Jaumann, R. H. Brown, J. M. Curchin, T. M. Hoefen, K. Stephan, C. M. Dalle Ore, B. J. Buratti, G. Filacchione, K. H. Baines, P. D. Nicholson, 2011b, The composition of Iapetus: Mapping Results from Cassini VIMS, Icarus, submitted.

Cruikshank, D.P., Roush, T.L., Bartholomew, M.J., Geballe, T.R., Pendleton, Y.J., White, S.M., Bell, J.F., Davies, J.K., Owen, T.C., de Bergh, C., Tholen, D.J., Bernstein, M.P., Brown, R.H., Tryka, K.A., Dalle Ore, C.M., 1998. The composition of Centaur 5145 Pholus. Icarus 135, 389-407. 
764

765

766

767

768

769

770

771

772

773

774

775

776

777

778

779

780

781

782

783

784

785

786

787

788

789

790

791

792

793

794

795

796

797

798

799

800

801

Cruikshank, D.P., Dalle Ore, C.M., Roush, T.L., Geballe, T.R., Owen, T.C., de Bergh, C., Cash, M.D., Hartmann, W.K., 2001. Constraints on the composition of Trojan Asteroid 624 Hektor. Icarus 153, 348-360.

Cruikshank, D.P., Owen, T.C., Dalle Ore, C., Geballe, T.R., Roush, T.L., de-Bergh, C., Sandford, S.A., Poulet, F., Benedix, G.K., Emery, J.P., 2005. A spectroscopic study of the surfaces of Saturn's large satellites: $\mathrm{H}_{2} \mathrm{O}$ ice, tholins, and minor constituents. Icarus 175, 268-283.

Cuzzi, J., R. Clark, G. Filacchione, R. French, R. Johnson, E. Marouf, L. Spilker, 2009. Ring particle composition and size distribution, In: Saturn after Cassini/Huygens, Springer, DOI 10.1007/978-1-40209215-2, pp. 459-509

Cuzzi, J.N., J. A. Burns, S. Charnoz, R. N. Clark, J. E. Colwell, L. Dones, L. W. Esposito, G. Filacchione, R. G. French, M. M. Hedman, S. Kempf, E. A. Marouf, C. D. Murray, P. D. Nicholson, C. C. Porco, J. Schmidt, M. R. Showalter, L. J. Spilker, J. N. Spitale, R. Srama, M. Sremcevic, M. S. Tiscareno, J. Weiss, 2010. An evolving view of Saturn's dynamic rings. Science, 327, 1470 - 1475.

Davidsson, B.J.R., Gutiérrez, P.J., Rickman, H., 2009. Physical properties of morphological units on Comet 9P/Tempel 1 derived from near-IR Deep Impact spectra. Icarus 201, 335-337.

Domingue, D., Verbiscer, A., 1997. Re-analysis of the solar phase curves of the icy Galilean satellites. Icarus $128,49-74$.

Domingue, D.L., Lockwood, G. and Thompson, D., 1995. Surface textural properties of icy satellites: A comparison between Europa and Rhea. Icarus 115, 228-249.

Domingue, D.L., Denevi, B.W., Ernst, C.M., Holsclaw, G.M., Izenber, N.R., McClintock, W.E., Murchie, S.L., Robinson, M.S., 2009. Regional color photometry of Mercury's surface. Lunar Planet. Sci. XL, 1301 (abstract).

Drossart, P. 1993. Optics on a fractal surface and the photometry of the regoliths. Planet. Space Sci. 41, 381393.

Emery, J.P., Burr, D.M., Cruikshank, D.P., Brown, R.H., Dalton, J. B., 2005. Near-infrared (0.8-4.0 um) spectroscopy of Mimas, Enceladus, Tethys, and Rhea. Astron. Astrophys. 435, 353-362.

Filacchione, G., Capaccioni, F., McCord, T.B., Coradini, A., Cerroni, P., Bellucci, G., Tosi, F., D’Aversa E., Formisano, V., Brown, R.H., Baines, K.H., Bibring, J.P., Buratti, B.J., Clark, R.N., Combes, M., Cruikshank, D.P., Drossart, P., Jaumann, R., Langevin, Y., Matson, D.L., Mennella, V., Nelson, R.M., Nicholson, P.D., Sicardy, B., Sotin, C., Hansen, G., Hibbitts, K., Showalter, M., Newman, S., 2007. Saturn's icy satellites investigated by Cassini-VIMS. I. Full-disk properties: $350-5100 \mathrm{~nm}$ reflectance spectra and phase curves. Icarus 186, 259-290.

Filacchione, G., Capaccioni, F., Clark, R.N., Cuzzi, J.N., Cruikshank, D.P., Coradini, A., Cerroni, P.,Nicholson, P.D., McCord, T.B., Brown, R.H., Buratti, B.J., Tosi, F., Nelson, R.M., Jaumann, R., Stephan K., 2010. Saturn's icy satellites investigated by Cassini-VIMS. II. Results at the end of nominal mission. Icarus $206,507-523$.

Grundy, W.M., 2009. Is the missing ultra-red material colorless ice? Icarus 199, 560-563.

Hapke, B., 1993. Theory of Reflectance and Emittance Spectroscopy, Topics in remote sensing: 3, Cambridge University Press, Cambridge, UK. 
802

803

804

805

806

807

808

809

810

811

812

813

814

815

816

817

818

819

820

821

822

823

824

825

826

827

828

829

830

831

832

833

834

835

836

837

838

839

840

Hapke, B., 2002. Bidirectional reflectance spectroscopy. 5. The coherent backscatter opposition effect and anisotropic scattering. Icarus 157, 523-534.

Hapke, B., 2008. Bidirectional reflectance spectroscopy. 6. Effects of porosity. Icarus 195, 918-926.

Hapke, B., Nelson, R., Smythe, W., 1998. The opposition effect of the moon: Coherent backscatter and shadow hiding. Icarus 133, 89-97.

Hapke, B., Shepard, M.K, Nelson, R.M., Smythe, W.D., Piatek, J.,L., 2009. A quantitative test of the ability of models based on the equation of radiative transfer to predict the bidirectional reflectance of a wellcharacterized medium. Icarus 199, 210-218.

Hudson, R.S. and Ostro S.J., 1999. Physical model of Asteroid 1620 Geographos from radar and optical data. Icarus $140,369-378$.

Iess, L., Rappaport, N.J., Tortora, P., Lunine, J., Armstrong, J.W., Asmar, S.W., Somenzi, L., Zingoni, F., 2007. Gravity field and interior of Rhea from Cassini data analysis. Icarus, 190, 585-593.

Khare, B.N., Sagan, C., Arakawa, E.T., Suits, R., Callcot, T.A., Williams, M.W., 1984. Optical constants of organic tholins produced in a simulated titanian atmosphere: From soft X-ray to microwave frequencies. Icarus 60, 127-137.

Khare, B.N., Thompson, W.R., Cheng, L., Chyba, C., Sagan, C., Arakawa, E.T., Meisse, C., Tuminello, P.S., 1993. Production and optical constraints of ice tholin from charged particle irradiation of (1:6) $\mathrm{C} 2 \mathrm{H} 6 / \mathrm{H} 2 \mathrm{O}$ at 77 K. Icarus 103, 290-300.

Lumme, K., and E. Bowell 1981. Radiative transfer in the surfaces of atmosphereless bodies. I. Theory. Astron. J. 86, 1694-1704.

MacKintosh, F.C., Sajeev, J., 1988. Coherent backscattering of light in the presence of time-reversalnoninvariant and parity-nonconserving media. Phys. Rev. B 37, 1884-1897.

Mallama, A., Wang, D., Howard, R.A., 2002. Photometry of Mercury from SOHO/LASCO and Earth. The phase function from 2 to 170 deg.. Icarus 155, 253-264.

Mallet, P., Guérin, C.A., Sentenac, A., 2005. Maxwell-Garnett mixing rule in the presence of multiple scattering: Derivation and accuracy. Phys. Rev. B 72, doi:10.1103/PhysRevB.72.014205

Mastrapa, R., Bernstein, M., Sandford, S., Roush, T., Cruikshank, D., Dalle Ore, C., 2008. Optical constants of amorphous and crystalline H2O-ice in the near infrared from 1.1 to $2.6 \mu \mathrm{m}$. Icarus 197, 307-320.

Mastrapa, R.M., Sandford, S.A., Roush, T.L., Cruikshank, D.P., Dalle Ore, C.M., 2009. Optica1

constants of amorphous and crystalline $\mathrm{H}_{2} 0-\mathrm{ice}: 2.5-22 \mu \mathrm{m}\left(4000-455 \mathrm{~cm}^{-1}\right)$ optical constants of $\mathrm{H}_{2} \mathrm{O}-\mathrm{ice}$. Astrophysical Journal 701, 1347-1356.

Maxwell-Garnett, J., 1904. Colours in metal glasses and in metallic films. Philosophical Transactions of the Royal Society of London. Series A 203 , 385-420.

McCord, T.B., Coradini, A., Hibbitts, C.A., Capaccioni, F., Hansen, G.B., Filacchione, G., Clark, R.N., Cerroni, P., Brown, R.H., Baines, K.H., Bellucci, G., Bibring, J.-P., Buratti, B.J., Bussoletti, E., Combes, M., Cruikshank, D.P., Drossart, P., Formisano, V., Jaumann, R., Langevin, Y., Matson, D.L., Nelson, R.M., Nicholson, P.D., Sicardy, B., Sotin, C., 2004. Cassini VIMS observations of the Galilean satellites including the VIMS calibration procedure. Icarus 172, 104-126. 
841

842

843

844

845

846

847

848

849

850

851

852

853

854

855

856

857

858

859

860

861

862

863

864

865

866

867

868

869

870

871

872

873

874

875

876

McDonald, G.D., Thompson, W.R., Heinrich, M., Khare, B.N., Sagan, C., 1994. Chemical investigation of Titan and Triton tholins. Icarus 108, 137-145.

McGuire, A., F. and Hapke, B. W., 1995. An experimental investigation of light scattering by large, irregular particles. Icarus 113, 134-155.

Mie, G., 1908. Beiträge zur Optik trüber Medien, speziell kolloidaler Metallösungen. Ann. Phys. 330, 377

Miller, E., Klein, G., Juergens, D., Mehaffey, K., Oseas, J., Garcia, R., Giandomenico, A., Irigoyen, B., Hickok, R., Rosing, D., Sobel, H., Bruce, C., Flamini, E., DeVidi, R., Reininger, F., Dami, M., Soufflot, A., Langevin, Y., Huntzinger, G., 1996. The Visual and Infrared Mapping Spectrometer for Cassini. Proc. SPIE 2803, 206-220.

Pitman, K.M., Buratti, B.J., Mosher, J.A., 2010. Disk-integrated bolometric Bond albedos and rotational light curves of saturnian satellites from Cassini Visual and Infrared Mapping Spectrometer

Poulet, F., Cuzzi, J.N., Cruikshank, D.P., Roush, T.L., Dalle Ore, C.M., 2002. Comparison between the Shkuratov and Hapke scattering theories for solid planetary surfaces: Application to the surface composition of two Centaurs. Icarus 160, 313-324.

Poulet, F., Cruickshank, D.P., Cuzzi, J.N., Roush, T.L., French, R.G., 2003. Compositions of Saturn's rings A, B, and C from high resolution near-infrared spectroscopic observations Astron. Astrophys 412, 305-316.

Roush, T.L., 1994. Charon: More than water ice? Icarus 108, 243-254.

Shepard, M.K., Helfenstein, P., 2007. A test of the Hapke photometric model. J. Geophys. Res. 112, doi: 10.1029/2005JE002625.

Shkuratov, Y., Starukhina, L., Hoffmann, H., Arnold, G., 1999a. A model of spectral albedo of particulate surfaces: Implications for optical properties of the Moon. Icarus 137, 245-246.

Shkuratov, Y., Kreslavsky, M.A., Ovcharenko, A.A., Stankevich, D.G., Zubko, E.S., Pieters, C., Arnold, G., 1999b. Opposition effect from Clementine data and mechanisms of backscatter. Icarus 141, 132-155.

Spencer, J.R., Denk, T., 2010. Formation of Iapetus' extreme albedo dichotomy by exogenically triggered thermal ice migration. Science $327,432$.

Thekaekara, M.P., 1973. Solar energy motion in space (SEMIS). In: Proc. Symp. Solar Radiation Meas. and Instrumentation, pp. 414-442. SEE N76-15973 06-92.

Thomas, P.C., Helfenstein, P., Veverka, J., Burns, J., Porco, C., Denk, T., Turtle, E., 2006. Sizes, shape, relaxation states and interior configurations of icy saturnian satellites. Bull. Am. Astron. Soc. 38, 621 (abstract).

Tosi, F., Turrini, D., Coradini, A., Filacchione, G., 2010. Probing the origin of the dark material on Iapetus. Mon. Not. R. Astron. Soc. 403, 1113-1130.

Verbiscer, A., and Veverka, J. 1989. Albedo dichotomy of Rhea: Hapke analysis of Voyager photometry. Icarus $82,336-353$. 
877 878

879 880

881

882

883

884

885

886

887

888

889

890

891

893

894

895

896

897

898

899

Warren, S.G., 1984. Optical constants of ice from the ultraviolet to the microwave. Applied Optics 23,12061225.

Warrel, J. and Davidsson, B.J.R., 2010. A Hapke model implementation for compositional analysis of VNIR spectra of Mercury. Icarus 209, 164-178.

Zubko, V.G., Mennella, V., Colangeli, L., Bussoletti, E., 1996. Optical constants of cosmic carbon analogue grains - I. Simulation of clustering by a modified continuous distribution of ellipsoids. Mon. Not. R. Astron. Soc. 282, 1321-1329.

\section{FIGURES CAPTIONS}

Figure 1. Schematic representations of two-components mixtures: areal (a), intimate (b) and intraparticle (c). In a) the circle represents the field of view of the observing instrument, while in c) the circle represents a single grain.

Figure 2. VIMS full-disk spectra of Rhea acquired at different phase angles, normalized at $1 \mu \mathrm{m}$. An offset is added for clarity. The spectrum at each phase angle is compared to the spectrum at $0.08^{\circ}$ (black curve). The leading fraction L.F. of each spectrum is reported.

Figure 3. Rhea's full-disk phase functions at various wavelengths. All the curves are normalized to the value at minimum phase angle $\left(0.08^{\circ}\right)$.

Figure 4. Real part $(n)$ of the refractive index for water ice and four organic contaminants:

hydrogenated amorphous carbon (ACH2), tholin from Khare et al. 1993, Titan tholin and Triton tholin. 
901

902

903

904

905

906

907

908

909

910

911

912

913

914

915

916

917

918

919

920

921

922

923

Figure 5. Imaginary part $(k)$ of the refractive index for water ice and four organic contaminants: hydrogenated amorphous carbon (ACH2), tholin from Khare et al. 1993, Titan tholin and Triton tholin.

Figure 6. Simulated spectra of areal mixtures of water ice and $\mathrm{ACH} 2$ (left panel) and of water ice and Tholin from Khare et al.1993 (right panel). The percentage of water ice is indicated. Spectra are normalized at $1 \mu \mathrm{m}$. Grain size is $50 \mu \mathrm{m}$.

Figure 7. Upper left panel: areal mixture best fit. It is obtained with water ice and Titan tholin. The percentages of water ice $(p)$ and contaminant $(p c)$ and the grain size are indicated. Observed spectrum is in red. Spectra are normalized at $1 \mu \mathrm{m}$. Upper right panel: intimate mixture best fit. It is obtained with water ice and Titan tholin. The percentages of water ice $(p)$ and contaminant $(p c)$ and the grain size are indicated. Observed spectrum is in red. Spectra are normalized at $1 \mu \mathrm{m}$. Bottom left panel: Summary plot of intraparticle mixtures fits. Rhea spectrum is the continuum line. All the mixtures are water ice + contaminant. Spectra are normalized at $1 \mu \mathrm{m}$. Grain sizes and compounds abundances are in Table 3 . Bottom right panel: intraparticle mixture best fit. It is obtained with water ice and Triton tholin. The percentages of water ice $(p)$ and contaminant $(p c)$ and the grain size are indicated. Observed spectrum is in red. Spectra are normalized at $1 \mu \mathrm{m}$.

Figure 8. Upper left panel: phase function fit residuals at each wavelength against the singlescattering albedo. Upper right panel: single-particle phase function at $20^{\circ}$ and $90^{\circ}$ ratio for each wavelength against the single-scattering albedo. Bottom left panel: opposition effect width against 
924 the single-scattering albedo. Bottom right panel: opposition effect amplitude against the single925 scattering albedo.

926

927 Figure 9. Rhea full-disk phase functions at wavelengths relative to increasing value of the single928 scattering albedo. Single-scattering albedo values and corresponding wavelengths are indicated. 929 Curves are normalized at minimum phase angle $\left(0.08^{\circ}\right)$.

930

931

932

933

934

935

936

937

938

939

940

941

942

943

944

Figure 10. Distribution of fitted $b$ (left panel) and $v$ (right panel) values respect to the single scattering albedo. $N$ represents how many times a certain value of the parameter is obtained in a given range of $w$ values. The $w$ range (0-1) is divided in intervals 0.1 wide.

Figure 11. Final absolute spectral fit at $g=90.2^{\circ}\left(p=0.996, p c=0.004, a_{m}=38 \mu m\right.$, intraparticle mixture). Isotropic single-particle phase function approximation has been removed and the correction due to roughness has been introduced. Observed spectrum is red.

Figure 12. Left panel: final absolute spectral fit at $g=2.1^{\circ}$. Right panel: final absolute spectral fit at $g=49.4^{\circ}$. The two simulations are obtained with an intraparticle mixture $\left(p=0.996, p c=0.004, a_{m}\right.$ $=38 \mu \mathrm{m})$. Isotropic single-particle phase function approximation has been removed and the correction due to roughness has been introduced. Observed spectrum is red. 
Highlights

949

$>$ Rhea's spectrum and phase function modeling. > Spectra from VIMS on board

950 Cassini spacecraft. >Intraparticle mixture of water ice and Triton tholin reproduces

951 observed reddening. > Determination of phase function parameters. >Dependence

952 of phase function parameters on single scattering albedo.

953 